\title{
Induction of CYP26A1 by Metabolites of Retinoic Acid: Evidence That CYP26A1 Is an Important Enzyme in the Elimination of Active Retinoids
}

\author{
Ariel R. Topletz, Sasmita Tripathy, Robert S. Foti, Jakob A. Shimshoni, Wendel L. Nelson, \\ and Nina Isoherranen \\ Departments of Pharmaceutics (A.R.T., S.T., J.A.S., N.I.) and Medicinal Chemistry (W.L.N.), University of Washington, Seattle, \\ Washington; and Department of Pharmacokinetics and Drug Metabolism, Amgen Inc., Seattle, Washington (R.S.F.)
}

Received November 9, 2014; accepted December 8, 2014

\begin{abstract}
All-trans-retinoic acid (atRA), the active metabolite of vitamin A, induces gene transcription via binding to nuclear retinoic acid receptors (RARs). The primary hydroxylated metabolites formed from atRA by CYP26A1, and the subsequent metabolite 4-oxoatRA, bind to RARs and potentially have biologic activity. Hence, CYP26A1, the main atRA hydroxylase, may function either to deplete bioactive retinoids or to form active metabolites. This study aimed to determine the role of CYP26A1 in modulating RAR activation via formation and elimination of active retinoids. After treatment of HepG2 cells with atRA, (4S)-OH-atRA, (4R)-OH-atRA, 4-oxo-atRA, and 18-OH-atRA, mRNAs of CYP26A1 and RAR $\beta$ were increased 300- to 3000-fold, with 4-oxo-atRA and atRA being the most potent inducers. However, $>60 \%$ of the $4-\mathrm{OH}$-atRA enantiomers were converted to 4-oxo-atRA in the first 12 hours of treatment, suggesting that the activity of the 4-OH-atRA was
\end{abstract}

due to 4-oxo-atRA. In human hepatocytes, atRA, 4-OH-atRA, and 4-oxo-atRA induced CYP26A1 and 4-oxo-atRA formation was observed from 4-OH-atRA. In HepG2 cells, 4-oxo-atRA formation was observed even in the absence of CYP26A1 activity and this formation was not inhibited by ketoconazole. In human liver microsomes, 4-oxo-atRA formation was supported by $\mathrm{NAD}^{+}$, suggesting that 4-oxo-atRA formation is mediated by a microsomal alcohol dehydrogenase. Although 4-oxo-atRA was not formed by CYP26A1, it was depleted by CYP26A1 $\left(K_{\mathrm{m}}=63 \mathrm{nM}\right.$ and intrinsic clearance $=90 \mu \mathrm{l} / \mathrm{min}$ per pmol $)$. Similarly, CYP26A1 depleted 18-OH-atRA and the 4-OH-atRA enantiomers. These data support the role of CYP26A1 to clear bioactive retinoids, and suggest that the enzyme forming active 4-oxo-atRA may be important in modulating retinoid action.

\section{Introduction}

All-trans-retinoic acid ( $a t \mathrm{RA})$, the active metabolite of vitamin A (retinol), is an essential signaling molecule during fetal development and adult life. Retinoid signaling plays a role in the maintenance of healthy skin, epithelia and the immune system (Napoli, 2012), regulation of insulin-stimulated glucose secretion (Kane et al., 2010), lipid homeostasis (Bonet et al., 2012), embryonic development (Duester, 2008), stem cell and neuronal differentiation (Maden, 2007; Gudas and Wagner, 2011), tissue repair and regeneration (Gudas, 2012) and spermatogenesis (Chung and Wolgemuth, 2004; Hogarth and Griswold, 2013). The biologic activity of $a t \mathrm{RA}$ is largely mediated by its binding to the nuclear ligand-activated retinoic acid receptors (RARs) and

\footnotetext{
This research was supported in part by the National Institutes of Health National Institute of General Medical Sciences [Grants R01-GM081569 and R01-GM111772]. Research reported in this publication was supported in part by the National Institutes of Health National Center for Advancing Translational Sciences through the Clinical and Translational Science Awards Program [Grant TL1-TR000422]. The content is solely the responsibility of the authors and does not necessarily represent the official views of the National Institutes of Health.

dx.doi.org/10.1124/mol.114.096784.
}

the peroxisome proliferator-activated receptor $\beta / \delta$ (Schug et al., 2007; Yu et al., 2012). As such, retinoid signaling in a specific cell depends on the expression of the nuclear receptors and the concentration of atRA within the cell (McBurney, 1993; Rhinn and Dollé, 2012). Hence, it is not surprising that RARs are expressed in a cell type-specific fashion (Chambon, 1996), and at RA concentrations vary considerably among retinoid responsive tissues (Kane et al., 2008).

The concentration of atRA is regulated by synthesis from retinol via retinaldehyde by alcohol and aldehyde dehydrogenases and elimination by cytochrome P450 (P450) enzymes (Napoli, 2012). The enzymes of the CYP26 family are primarily responsible for atRA clearance forming the hydroxylated metabolites 4-OH-atRA, 18-OH-atRA (Fig. 1), and perhaps 16-OH-atRA (White et al., 1997, 2000; Chithalen et al., 2002; Thatcher et al., 2011; Topletz et al., 2012). CYP26A1 is the predominant atRA hydroxylase in the adult human liver (Thatcher et al., 2010; Topletz et al., 2012), and it is highly inducible by atRA via RAR-mediated mechanism (White et al., 1997; Yamamoto et al., 2000; Loudig et al., 2005; Tay et al., 2010) leading to autoinduction of CYP26A1 by atRA. This autoinduction allows

ABBREVIATIONS: atRA, all-trans-retinoic acid; AUC, area under the concentration curve; CRABP, cellular retinoic acid binding protein; HPLC, high-performance liquid chromatography; KPi, potassium phosphate; LC, liquid chromatography; MS/MS, tandem mass spectrometry; P450, cytochrome P450; RA, retinoic acid; RAR, retinoic acid receptor. 


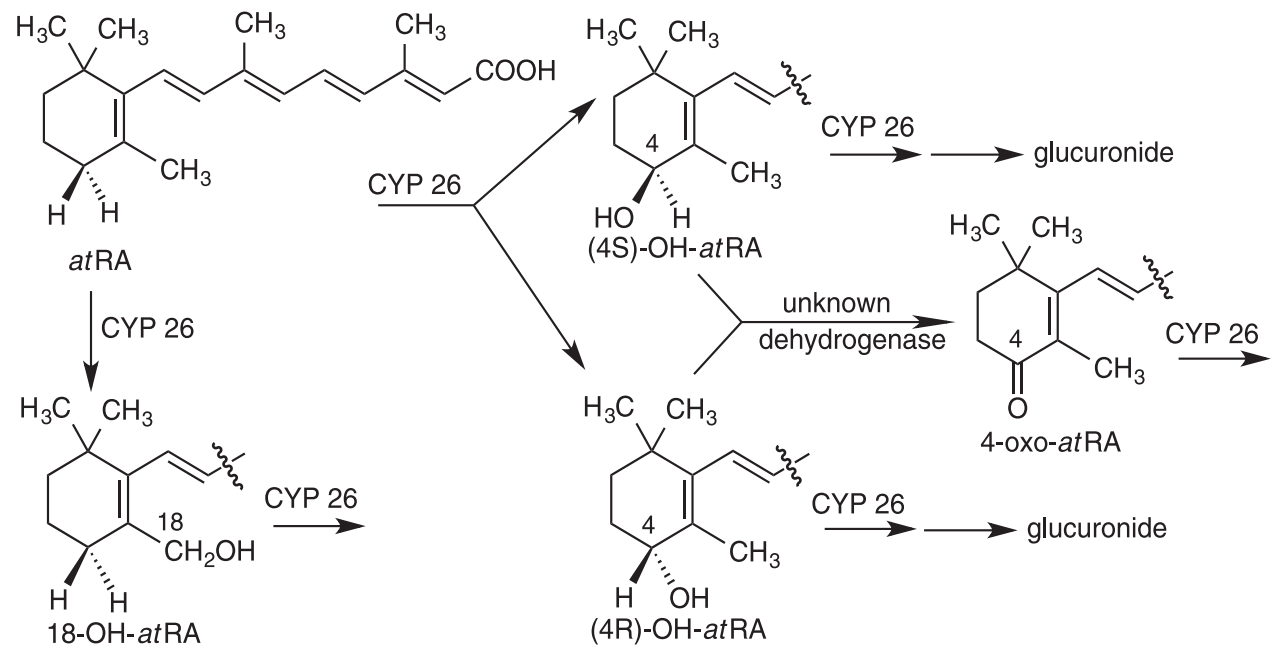

Fig. 1. Chemical structures of atRA, 4-oxoatRA, (4S)-OH-atRA, (4R)-OH-atRA, and 18-OH-atRA and the proposed metabolic pathways for atRA and its metabolites. The specific stereochemistry and the localization of the hydroxylations are shown together with the known enzymatic pathways for the metabolism of atRA and its metabolites. The quantitative importance of the indicated enzymes is not known. for efficient feedback for at RA regulating its own clearance but also suggests that atRA will induce the formation of potentially active metabolites by CYP26A1. The primary hydroxylation products 4-OH-atRA, 18-OH-atRA, and the sequential metabolite 4-oxo-at RA from 4-OH-atRA bind to RARs including RAR $\alpha$ (Idres et al., 2002). Since CYP26A1 forms metabolites that have RAR binding affinity, it is possible that CYP26A1 may play a role in forming metabolites with specific retinoid activity. However, the biologic role of atRA metabolites is largely unknown. Although genetic studies have shown that the developmental malformations of CYP26A1 $1^{-/-}$mice are mainly due to excess $a t \mathrm{RA}$ and not to the lack of formation of an active metabolite (Niederreither et al., 2002), it is not known whether the metabolites are important for specific processes later in life.

Of the metabolites from atRA, 4-oxo-atRA is the most studied and the most potent in terms of RAR activation, having a 3-fold lower $\mathrm{EC}_{50}$ than atRA toward RAR $\alpha$ (Idres et al., 2002). 4-Oxo-atRA is also detected in human plasma, suggesting that it may have biologic significance (Muindi et al., 1992b; Arnold et al., 2012). The potential biologic importance of 4-oxo-atRA is supported by studies that show that 4-oxo-atRA is teratogenic in mice and zebrafish with similar potency as atRA itself (Creech Kraft et al., 1989; Herrmann, 1995). In human skin cells, 4-oxo-atRA displays strong transcriptional regulatory activity (Baron et al., 2005). In Xenopus embryos, 4-oxo-atRA modulates positional specification (Pijnappel et al., 1993). Finally, 4-oxo-atRA induces spermatogenesis in vitamin A-deficient mice (Gaemers et al., 1996). Whether 4-oxo-atRA is formed by CYP26 enzymes is not clear, and the processes that regulate the homeostasis of 4-oxo-atRA are not well understood. As such, the importance of 4-oxo-atRA in retinoid biology is poorly defined.

This study aimed to determine whether CYP26A1 is induced by oxidated metabolites of atRA, and whether CYP26A1 forms and clears the oxidated metabolites including 4-oxo-atRA. The results of this study show that oxidated atRA metabolites are substrates of CYP26A1, and they induce their own clearance by CYP26A1. However, formation of 4-oxo-atRA, the metabolite with high RAR activation potency, is not mediated by CYP26A1 in cells or in the human liver. Taken together, these results support the conclusion that CYP26A1 functions primarily to eliminate bioactive retinoids, and that additional enzymes are required to form the bioactive metabolites of atRA such as 4-oxo-atRA.

\section{Materials and Methods}

Chemicals and Reagents. atRA, ketoconazole, fomepizole (4methylpyrazole), cimetidine, testosterone, and carbenoxolone were purchased from Sigma-Aldrich (St. Louis, MO). Talarozole was purchased from MedChem Express (Monmouth, NJ). 4-Oxo-atRA- $d_{3}$ (3 deuteriums at the C-18 position) and $a t \mathrm{RA}-d_{5}$ (2 deuteriums at the C-4 and 3 deuteriums at the $\mathrm{C}-18$ positions) were purchased from Toronto Research Chemicals (North York, Ontario, Canada). (4R)-OH-atRA, (4S)-OH-atRA, 4-oxo-atRA, and 18-OH-atRA (Fig. 1) were synthesized as previously described (Shimshoni et al., 2012; Topletz et al., 2012). Optima-grade water, optima-grade acetonitrile, ethanol, and ethyl acetate were purchased from Fisher Scientific (Pittsburgh, PA). Biologic DNAase/ RNAase-free water was purchased from Qiagen (Valencia, CA).

Quantitative Analysis of Retinoids. atRA, 4-oxo-atRA, $(4 R)-\mathrm{OH}-$ $a t \mathrm{RA},(4 S)-\mathrm{OH}-a t \mathrm{RA}$, and 18-OH-atRA (Fig. 1) were extracted from cell and medium samples and from incubations using ethyl acetate under red light as previously described (Thatcher et al., 2010; Topletz et al., 2012). The solvent was evaporated under a nitrogen stream, and the dry residue was reconstituted in $100 \mu \mathrm{l}$ acetonitrile for analysis by highperformance liquid chromatography (HPLC) coupled to tandem mass spectrometry (MS/MS)

Analytes were separated using an Agilent 1290 Infinity UHPLC system, an Agilent Zorbax C18 column (3.5 $\mu \mathrm{m}, 2.1 \mathrm{~mm} \times 100 \mathrm{~mm}$ ), and a mobile phase flow of $0.2 \mathrm{ml} / \mathrm{min}$ and a linear 30 -minute gradient with initial mobile phase of $10: 90$ acetonitrile/aqueous $0.1 \%$ formic acid increasing to $90: 10$ acetonitrile/aqueous $0.1 \%$ formic acid (held for 2 minutes) followed by a re-equilibration back to initial mobile phase conditions over 3 minutes. The analytes were detected using an AB Sciex API 5500 Q/LIT mass spectrometer (AB Sciex, Framingham, MA) using negative ion electrospray detection, with the following: declustering potentials of $-80 \mathrm{~V}$ (4-OH-atRA and 18-OH-atRA), $-90 \mathrm{~V}$ (4-oxo- $a t$ RA- $\left.d_{3}\right),-140 \mathrm{~V}$ ( $\left.a t \mathrm{RA}\right),-100 \mathrm{~V}$ ( $a t \mathrm{RA}-d_{5}$ ), and -95 V (4-oxo-atRA and 4-oxo-13-cisRA); collision energies of $-28 \mathrm{eV}$ (4-OH-atRA and 18-OH-atRA), $-25 \mathrm{eV}$ (4-oxo- $\left.a t \mathrm{RA}-d_{3}\right),-40 \mathrm{eV}$ (atRA- $\left.d_{5}\right)$, and $-22 \mathrm{eV}$ (atRA, 4-oxo-atRA, and 4-oxo-13-cisRA); and collision exit potentials of $-7 \mathrm{~V}$ (atRA, 4-OH-atRA, and 18-OH-atRA), $-5 \mathrm{~V}$ (4-oxo-atRA and 4-oxo-13-cisRA), $-16 \mathrm{~V}$ ( $\left.a t \mathrm{RA}-d_{5}\right)$, and $-10 \mathrm{~V}$ (4-oxo- $\left.a t \mathrm{RA}-d_{3}\right)$. The parent-fragment MS/MS transitions of $m / z 299 \rightarrow 255 \mathrm{Da}$ (atRA), $304 \rightarrow 260 \mathrm{Da}\left(a t \mathrm{RA}-d_{5}\right), 315 \rightarrow 253 \mathrm{Da}$ (4-OH- $a t \mathrm{RA}$ and 18-OH- $\left.a t \mathrm{RA}\right)$, $313 \rightarrow 269 \mathrm{Da}$ (4-oxo-atRA and 4-oxo-13-cisRA), and $316 \rightarrow 272 \mathrm{Da}$ (4-oxo-atRA- $d_{3}$ ) were monitored. The lower limits of quantification were $3.1 \mathrm{nM}$ for $4-\mathrm{OH}-a t \mathrm{RA}, 0.3 \mathrm{nM}$ for $4-\mathrm{oxo}-a t \mathrm{RA}$, and $6.3 \mathrm{nM}$ for $18-\mathrm{OH}-$ $a t$ RA. Standard curves were reproducible within $15 \%$ of each other from three separate extractions on three different days of analysis.

Cell Culture and mRNA Analysis. The HepG2 cells were obtained and cultured in a humidified incubator at $37^{\circ} \mathrm{C}$ as previously described (Tay et al., 2010) under an atmosphere of 5\% carbon dioxide 
using ATCC Minimum Essential Medium (American Type Culture Collection, Manassas, VA) with Earle's balanced salt solution supplemented with $10 \%$ fetal bovine serum and $0.5 \%$ penicillin as the growth medium (Tay et al., 2010). For atRA and atRA metabolite treatments, cells were plated into six-well plates $\left(10^{6}\right.$ cells per well) in $2 \mathrm{ml}$ medium/well and cells were allowed to adhere for 24 hours prior to treatments. The cells were treated for 48 hours in $2 \mathrm{ml}$ medium, changing the medium (+ inducer) at 24 hours. The treatments included atRA, 4-oxo-atRA, (4R)-OH-atRA，(4S)-OH-atRA， or 18-OH-atRA at concentrations of $1 \mathrm{nM}$ to $1 \mu \mathrm{M}$ or $0.1 \% \mathrm{EtOH}$ as a vehicle control. All treatments were performed in triplicate. At the completion of each treatment, the medium in each well was aspirated and cells were harvested for mRNA extraction. The time course of CYP26A1 and RAR $\beta$ induction in HepG2 cells was characterized after a $1-\mu \mathrm{M}$ treatment with $a t \mathrm{RA}$. The cells were cultured as above but cells were harvested at $6,12,24$, and 48 hours after treatment for mRNA extraction.

Pooled cryopreserved human hepatocytes from two male donors and one female donor were obtained from Life Technologies (Grand Island, NY). Hepatocytes were thawed at $37^{\circ} \mathrm{C}$, placed into plating media (InVitroGro CP medium plus Torpedo antibiotic mix; Bioreclamation IVT, Baltimore, MD), and cell viability was confirmed. Hepatocytes were plated at $10^{6}$ live cells per well in six-well collagen type I-coated plates (Becton Dickinson, Franklin Lakes, NJ) and were allowed to attach for approximately 8 hours. The plating media was then replaced with Williams E media containing primary hepatocyte maintenance supplements (Life Technologies) and cells were treated with atRA, 4-OH-atRA, or 4-oxo-atRA each at 100,500 , and $1000 \mathrm{nM}$ or ethanol as a vehicle control for 2 hours. The short, 2 -hour treatment period was chosen to minimize retinoid depletion and metabolism in the treatments. Immediately after addition of the retinoids and at the end of the 2-hour induction period, an aliquot of the media was collected for retinoid concentration measurements as described above. At the end of the 2hour induction period, the cells were collected for mRNA measurements.

For mRNA extraction, $300 \mu \mathrm{l}$ of TRI reagent (Invitrogen, Grand Island, NY) was added to each well and mRNA was extracted according to the manufacturer's recommendations. Total RNA was quantified using the Nanodrop 2000c Spectrophotometer (Thermo Fisher Scientific, Waltham, MA) and RNA quality was determined by gel electrophoresis. cDNA was generated from $1 \mu \mathrm{g}$ mRNA using TaqMan gene expression reagents (Applied Biosystems, Carlsbad, CA). Reverse-transcription polymerase chain reaction was used to quantify CYP26A1 and RAR $\beta$ mRNA (StepOnePlus; Applied Biosystems) as previously described (Tay et al., 2010). TaqMan real-time gene expression master mix and polymerase chain reaction primers and fluorescent probes were obtained from Applied Biosystems (Foster City, CA). Probes were labeled with the $5^{\prime}$-reporter dye 5 -carboxyfluorescein and a nonfluorescent black hole quencher on the $3^{\prime}$ end. Primer and probe pairs used included the following: CYP26A1 (Hs00175627_m1, FAM), glyceraldehyde-3-phosphate dehydrogenase (Hs99999905_m1, VIC), RAR $\alpha$ (Hs00940446_m1, FAM), RAR $\beta_{-}$(Hs00233407_m1, FAM), RAR $\gamma$ (Hs00171273_m1, FAM), cellular retinoic acid binding protein (CRABP)-I (Hs00171635_m1, FAM), and CRABP-II (Hs00275636_m1, FAM). Glyceraldehyde-3-phosphate dehydrogenase was used as the housekeeping gene and all assays were done as multiplexes. All triplicate samples were analyzed in duplicate. Changes in target mRNA were measured using relative quantification (fold-difference) and the $\Delta \Delta$ cycle threshold method (Tay et al., 2010) using GraphPad Prism software (version 5; GraphPad Software Inc., La Jolla, CA).

RAR Reporter Assays. Human $\operatorname{RAR} \alpha, \operatorname{RAR} \beta$, and RAR $\gamma$ reporter assays (Indigo Biosciences, State College, PA) were used to determine the ability of atRA, 4-OH-atRA, and 4-oxo-atRA to act as agonists of human RARs. In brief, reporter cells were rapidly thawed into prewarmed cell recovery media and plated into a 96-well assay plate (100 $\mu \mathrm{l}$ reporter cell suspension per well). atRA and its metabolites (0-2 $\mu \mathrm{M}$, final concentration in compound screening media) were then added to the assay plate. Each reporter assay plate was placed in a humidified incubator $\left(37^{\circ} \mathrm{C} / 5 \% \mathrm{CO}_{2}\right)$ for approximately 20 hours.
Assay plates were then removed from the incubator and the contents aspirated from each well. Luciferase detection reagent $(100 \mu \mathrm{l})$ was added to each well and the plates allowed to rest at room temperature for 30 minutes. Luminescence was then measured on a Tecan Safire2 plate reader (Tecan, San Jose, CA) set to quantify luminescence using a read time of 500 milliseconds per well.

Retinoid Depletion and Subsequent Metabolite Formation in HepG2 Cells. Depletion of atRA, 4-oxo-atRA, (4R)-OH-atRA, (4S)$\mathrm{OH}-a t \mathrm{RA}$, or 18-OH-atRA and formation of subsequent metabolites from these substrates in HepG2 cells was measured in the media and in the cells to assess the exposure of the cells to the bioactive retinoids. HepG2 cells were plated and cultured as described above. Cells were treated with atRA, 4-oxo-atRA, (4R)-OH-atRA, $(4 S)-\mathrm{OH}-a t \mathrm{RA}$, or 18-OH- $a t \mathrm{RA}$ at a concentration of $1 \mu \mathrm{M}$ (nominal initial concentration in the medium) for 48 hours. The medium (+ inducer) was changed after 24 hours to mimic the design of the mRNA induction experiments. At $0,1,4,8,12,24$, and 48 hours, $1 \mathrm{ml}$ of the medium was removed, an internal standard ( $a t \mathrm{RA}-d_{5} ; 20 \mu \mathrm{l}$ of a $2.5 \mu \mathrm{M}$ stock solution) was added, and samples were extracted using ethyl acetate as described above. As controls, 4-oxo-atRA, (4R)-OH-atRA, or $(4 S)-\mathrm{OH}-a t \mathrm{RA}(1 \mu \mathrm{M})$ were incubated in $1 \mathrm{ml}$ of medium each for 4 hours in a $37^{\circ} \mathrm{C}$ oscillating water bath and the media were analyzed for substrate depletion and metabolite formation. Cells were collected at 1, 4, 8, and 12 hours after the beginning of the treatments by addition of $1 \mathrm{ml}$ acetonitrile on the cells in each well. One milliliter of potassium phosphate (KPi) buffer (100 mM, pH 7.4) was added to the acetonitrile phase to facilitate separation of the organic and aqueous layers, an internal standard (4-oxo-RA- $d_{3} ; 20 \mu \mathrm{l}$ of a $2.5 \mu \mathrm{M}$ stock solution) was added, and samples were extracted with ethyl acetate as previously described. The amounts of analytes in the extract were determined by liquid chromatography (LC)-MS/MS and the retinoid concentrations in the cells were calculated using a total intercellular volume of $1 \mu \mathrm{l} / 10^{6}$ HepG2 cells (Kewn et al., 2000).

Measurement of CYP26A1 Activity in HepG2 Cells. The formation of 4-OH-atRA- $d_{4}$ and 4-oxo-atRA- $d_{3}$ from $a t \mathrm{RA}-d_{5}$ was used to determine CYP26A1 activity and induction during treatment of HepG2 cells with atRA. HepG2 cells were treated for 48 hours with atRA ( $1 \mu \mathrm{M}$ final concentration in the medium). The medium containing atRA was changed at 24 hours. At $0.5,6,12,18,24,36$, and 48 hours, atRA-d $d_{5}(100 \mathrm{nM})$ was added to each well $(3 \mathrm{ml})$ and formation of 4-OH-atRA- $d_{4}$ and 4-oxo- $a t \mathrm{RA}-d_{3}$ after a 1-hour incubation was measured. All incubations were conducted in triplicate. An initial aliquot of the medium $(1 \mathrm{ml})$ was removed 30 seconds after $a t$ RA- $d_{5}$ addition as a control; after 1 hour at $37^{\circ} \mathrm{C}$, a second aliquot of medium $(1 \mathrm{ml})$ was removed from each well. 4-Oxo-13-cisRA $(20 \mu \mathrm{l}$ of a $2.5 \mu \mathrm{M}$ stock solution in EtOH) was added to each sample as an internal standard, and samples were extracted using $3 \mathrm{ml}$ ethyl acetate and analyzed using HPLC-MS/MS as described above. 4-Oxo-atRA- $d_{3}$ was quantified using a reference material. Due to the lack of a standard, the relative change in formation of $4-\mathrm{OH}-a t \mathrm{RA}-d_{4}$ was determined from the analyte to internal standard peak height ratio. Standard curves were constructed for $a t \mathrm{RA}-d_{5}$ and 4-oxo-atRA- $d_{3}$. The rate of 4-oxo-atRA- $d_{3}$ formation from $4-\mathrm{OH}-a t \mathrm{RA}-d_{4}$ was assumed to remain constant during treatment.

Characterization of 4-Oxo-atRA Formation from 4-OH-atRA in HepG2 cells. To determine whether formation of 4-oxo-atRA is predominantly P450-mediated in HepG2 cells, the HepG2 cells were incubated with atRA or 4-OH-atRA ( $1 \mu \mathrm{M}$ in the medium with $0.1 \%$ $\mathrm{EtOH})$ for 24 hours in the presence and absence of ketoconazole (10 $\mu \mathrm{M}$ in the medium with $0.1 \% \mathrm{EtOH}$ ), a potent pan-P450 inhibitor (Thatcher et al., 2011). In addition, HepG2 cells were incubated in the presence of atRA and 4-OH-atRA with vehicle or alcohol dehydrogenase inhibitors 4-methylpyrazole $(250 \mu \mathrm{M})$ and cimetidine $(250 \mu \mathrm{M})$, the aldo-keto reductase inhibitor testosterone $(25 \mu \mathrm{M})$, the retinol dehydrogenase inhibitor carbenoxolone (25 and $50 \mu \mathrm{M})$, and in the presence of atRA with talarozole (a selective CYP26 inhibitor; $1 \mu \mathrm{M}$ ). All treatments were performed in triplicate. An initial aliquot of the medium $(0.5 \mathrm{ml})$ was taken before medium was added to each well 

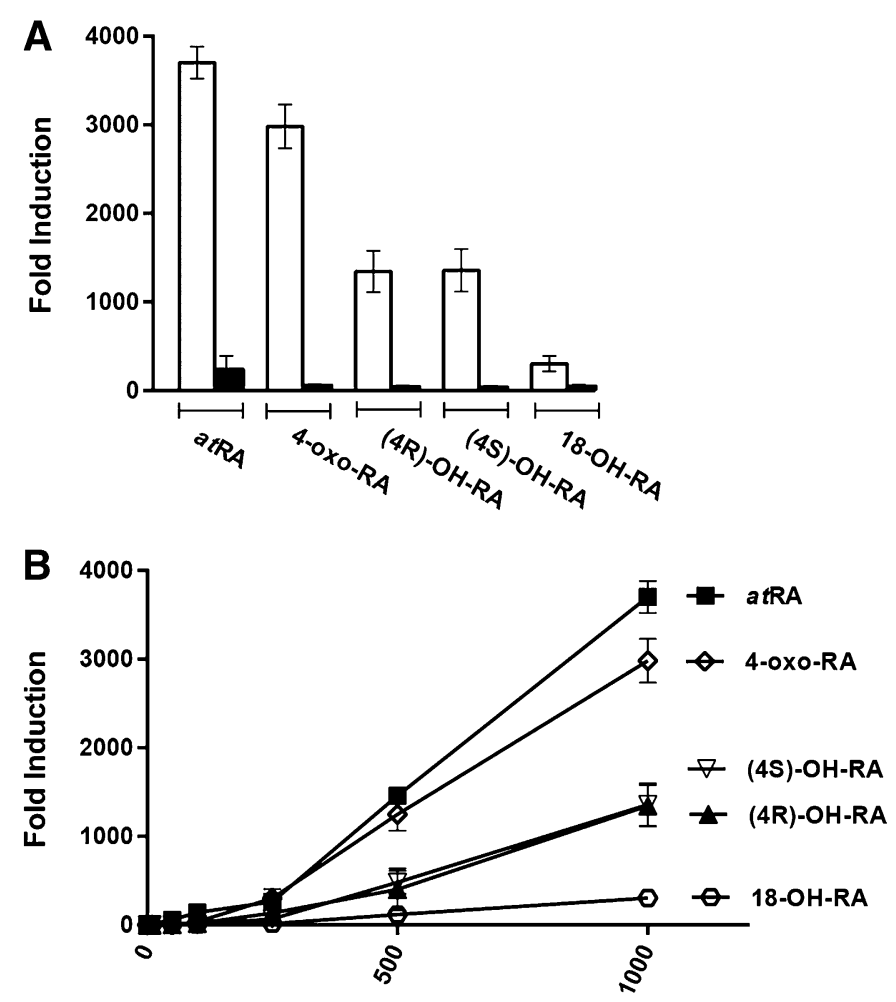

Concentration (nM)

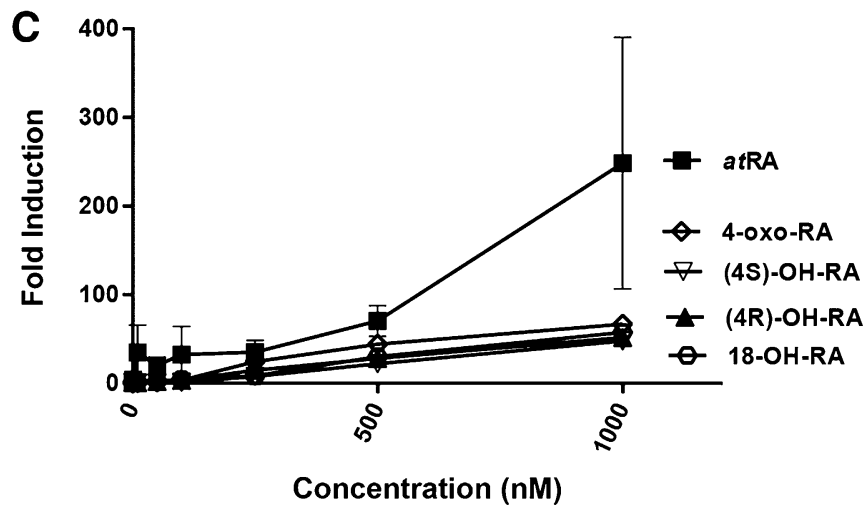

Fig. 2. Induction of CYP26A1, and RAR $\beta$ in HepG2 cells treated with at RA and its metabolites. (A) Fold induction of CYP26A1 (white bars) and RAR $\beta$ (black bars) mRNA by atRA, 4-oxo-at RA, (4R)-OH-atRA, (4S)-OH-atRA, and 18-OH- atRA after a $1 \mu \mathrm{M}$ treatment per 48 hours in HepG2 cells. (B and C). The induction of CYP26A1 (B) and RAR $\beta$ (C) mRNA by $a t R A, 4-o x o-a t R A$, $(4 R)-\mathrm{OH}-a t \mathrm{RA},(4 S)-\mathrm{OH}-a t \mathrm{RA}$, and 18-OH-atRA in HepG2 cells as a function of inducer concentration. Cells were treated for 48 hours at seven concentrations (ranging from $1 \mathrm{nM}$ to $1 \mu \mathrm{M}$ ) of each substrate, changing media after 24 hours. EtOH-treated cells served as the vehicle control and glyceraldehyde-3-phosphate dehydrogenase was used as a housekeeping gene for reverse-transcription polymerase chain reaction analysis.

to quantify the initial substrate concentrations per well. After 24 hours, a second aliquot of the medium $(0.5 \mathrm{ml})$ was removed and extracted with ethyl acetate. Standard curves were constructed for atRA, 4-oxo-atRA, and 4-OH-atRA with an internal standard of 4-oxo-atRA- $d_{3}$ ( $20 \mu \mathrm{l}$ of a $2.5 \mu \mathrm{M}$ stock solution). All samples were separated and analyzed for 4-OH-atRA and 4-oxo-atRA formation using the above-described HPLC-MS/MS method.

Characterization of 4-Oxo-RA and 18-OH-RA as CYP26A1 Substrates. The metabolism of 4-oxo-atRA and 18-OH-atRA by CYP26A1 was evaluated by substrate depletion using a similar protocol as previously described for characterization of 4-OH-atRA depletion (Shimshoni et al., 2012; Topletz et al., 2012). The Bac-to-Bac Baculovirus

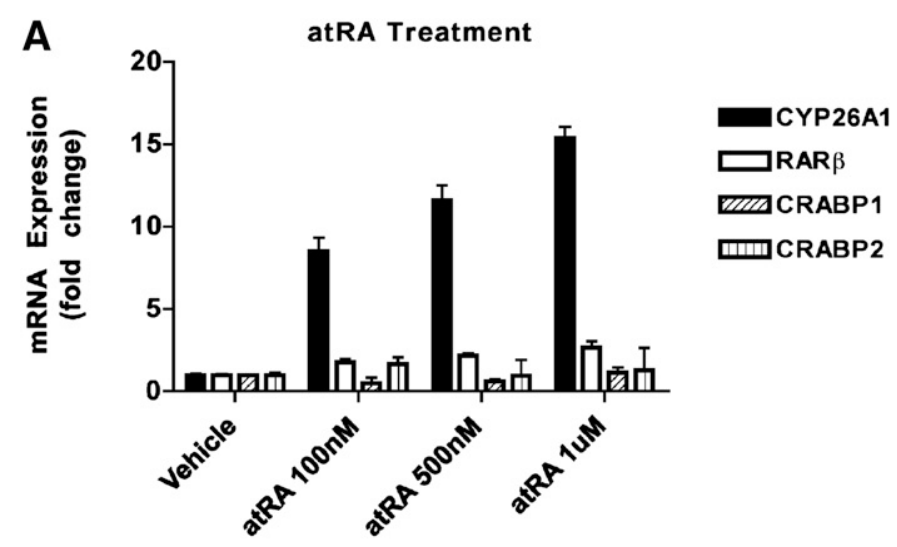

B

4-OH-atRA Treatment

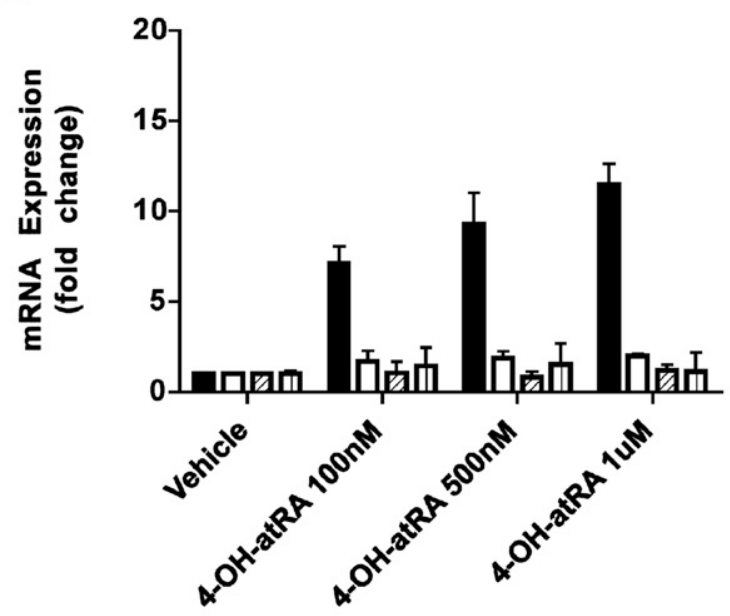

C

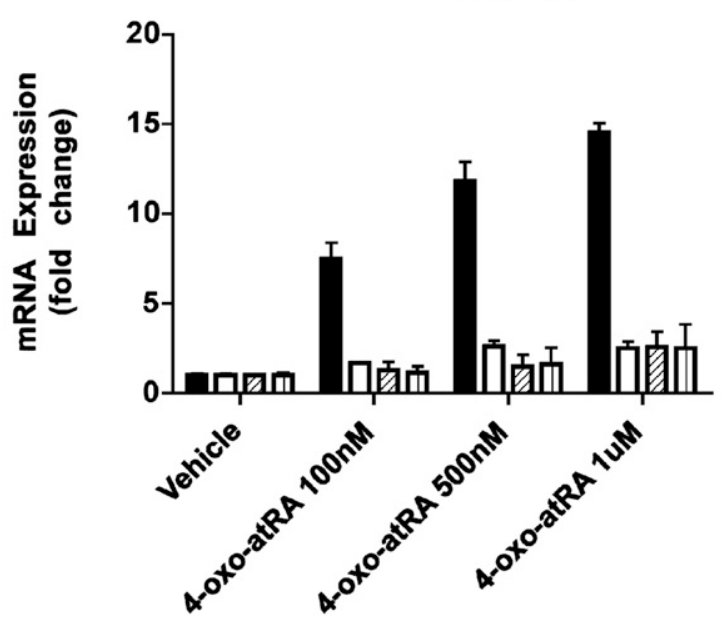

Fig. 3. Induction of CYP26A1, RAR $\beta$, CRABP-I, and CRABP-II in human hepatocytes. The induction of CYP26A1, RAR $\beta$, CRABP-I, and CRABP-II mRNA after treatment of human hepatocytes for 2 hours with 100,500 , and $1000 \mathrm{nM}$ of atRA (A), 4-OH-atRA (B), and 4-oxo-atRA (C) is shown compared with vehicle-treated cells.

Expression System (Invitrogen) with Sf9 insect cells was used to produce His-tagged CYP26A1 protein according to the manufacturer's instructions using Sf-900 II SFM liquid media (Invitrogen) supplemented with $2.5 \%$ fetal bovine serum. During protein expression, ferric citrate $(0.2 \mathrm{mM})$ and $\delta$-aminolevulinic acid $(0.3 \mathrm{mM})$ were added to the media 24 hours after infection to facilitate heme synthesis. The cells were harvested 72 hours after infection, washed once in phosphate-buffered 

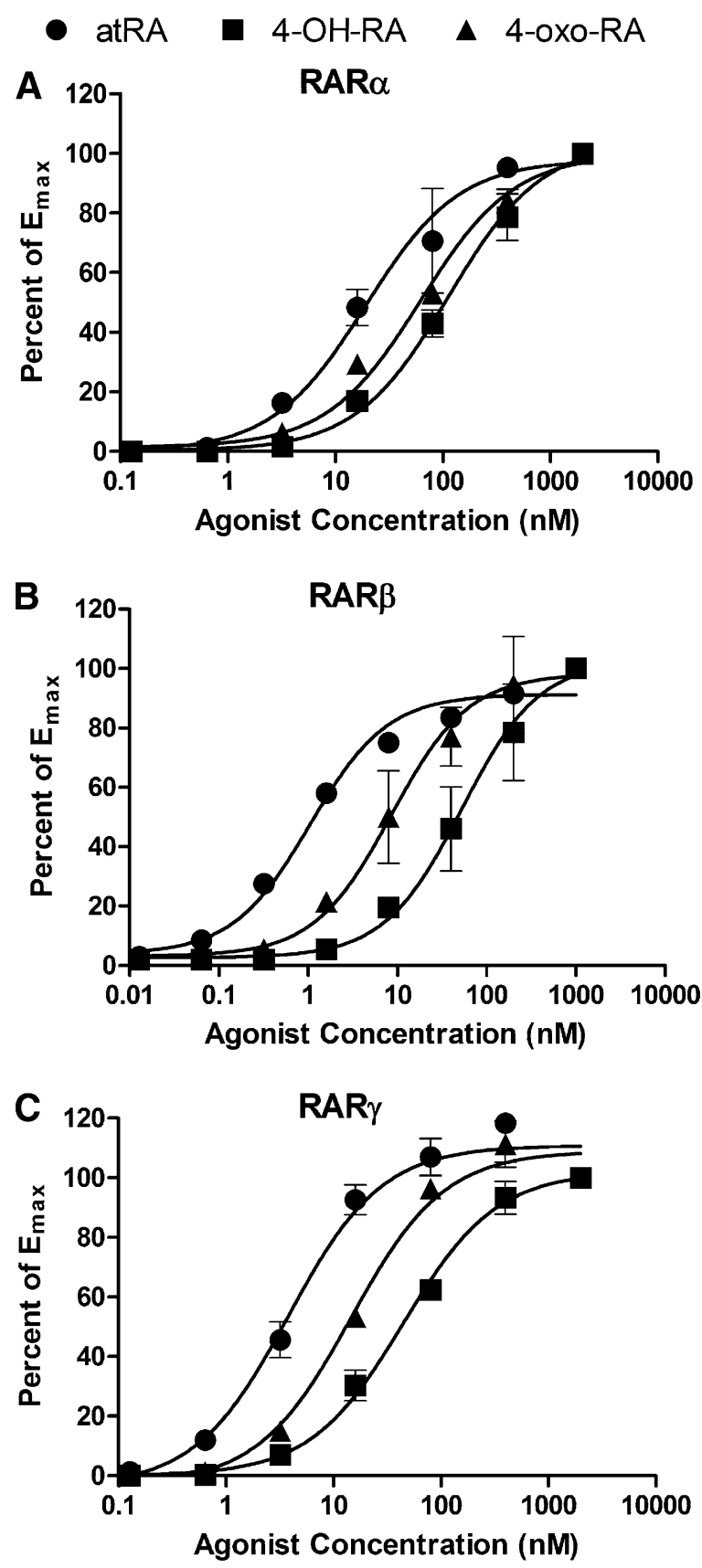

Fig. 4. Activation of $\operatorname{RAR} \alpha, \operatorname{RAR} \beta$ and RAR $\gamma$ by $a t \mathrm{RA}, 4-\mathrm{OH}-a t \mathrm{RA}$, and 4-oxo-atRA. The activation of $\operatorname{RAR} \alpha, \operatorname{RAR} \beta$, and $\operatorname{RAR} \gamma$ was determined using reporter assays at ligand concentrations between 0 and $2000 \mathrm{nM}$.

saline with $1 \mathrm{mM}$ phenylmethanesulphonylfluoride, pelleted, and stored at $-80^{\circ} \mathrm{C}$. Insect cell microsomes were prepared by standard ultracentrifugation at $120,000 \mathrm{~g}$ and supplemented with $\mathrm{P} 450$ reductase prior to incubations. The $\mathrm{P} 450$ content of the final microsomal preparation was determined by the CO-difference spectrum.

For quantification of the substrates, standard curves for each substrate were prepared in $100 \mathrm{mM} \mathrm{KPi}$ buffer ( $\mathrm{pH}$ 7.4) containing CYP26A1 (2 pmol/ml enzyme and $4 \mathrm{pmol} / \mathrm{ml}$ rat reductase) as previously described (Lutz et al., 2009; Thatcher et al., 2011). In brief, incubations containing substrate, $2 \mathrm{pmol} / \mathrm{ml} \mathrm{CYP} 26 \mathrm{~A} 1$ in the endoplasmic reticulum membrane, and $4 \mathrm{pmol} / \mathrm{ml}$ purified rat reductase in $100 \mathrm{mM}$ KPi buffer ( $\mathrm{pH}$ 7.4) were preincubated for 5 minutes at $37^{\circ} \mathrm{C}$ before the addition of NADPH (final concentration of $1 \mathrm{mM}$ ) to initiate the reaction. Each sample initially contained a total volume of $2 \mathrm{ml}$, and 4-oxo-at $\mathrm{RA}$ and 18-OH-atRA were added at concentrations ranging from 5 to $150 \mathrm{nM}$. At $0.5,1,2$, and 5 minutes, aliquots of $0.5 \mathrm{ml}$ were collected and immediately quenched in a test tube containing $3 \mathrm{ml}$ ice-cold ethyl acetate. For the final time point, ethyl acetate was added directly to the incubation vial. The internal standard 4-oxo- $a t \mathrm{RA}-d_{3}(20 \mu \mathrm{l}$ of a $2.5 \mu \mathrm{M}$ stock solution in EtOH) was added, and samples were extracted as described above. All incubations were conducted in duplicate and 4-oxo-atRA and 18-OH-atRA were quantified using standard curves.

Retinoids from the in vitro incubations were analyzed using LC-MS/MS as described above for cell culture experiments except that analytes were detected using positive ion electrospray mass spectrometry, with the declustering potentials of $80 \mathrm{~V}$, collision energies of $20 \mathrm{eV}$ (18-OH-atRA), $35 \mathrm{eV}$ (4-oxo-atRA- $d_{3}$ ), and $37 \mathrm{eV}$ (4-oxo-atRA), and collision exit potentials of $13 \mathrm{~V}$ (18-OH-RA and 4-oxo-atRA) and 2 (4-oxo- $\left.a t \mathrm{RA}-d_{3}\right)$. The parent-fragment MS/MS transitions of $m / z 317 \rightarrow 253 \mathrm{Da}$ (18-OH-atRA), $315 \rightarrow 121 \mathrm{Da}$ (4-oxo-atRA), and $300 \rightarrow 226 \mathrm{Da}$ (4-oxo-atRA- $d_{3}$ ) were monitored.

Formation of 4-Oxo-atRA from 4-OH-atRA in Human Liver Microsomes. Human liver microsomes $(0.2 \mathrm{mg} / \mathrm{ml})$ were incubated with 4-OH-atRA $(500 \mathrm{nM})$ and $\mathrm{NADPH}, \mathrm{NADP}^{+}$, or $\mathrm{NAD}^{+}$(each at $2 \mathrm{mM}$ ) in $100 \mathrm{mM} \mathrm{KPi}$ buffer $\mathrm{pH}$ 7.4. In addition, 4-OH-atRA was incubated with human liver microsomes in the presence and absence of talarozole (a CYP26A1-specific inhibitor; $1 \mu \mathrm{M}$ ) and ketoconazole (a pan-P450 inhibitor; $10 \mu \mathrm{M}$ ) and with NADPH as a cofactor. After a 5-minute preincubation, the reactions were initiated with the addition of cofactor and incubated for 30 minutes. At 30 minutes, the reactions were quenched with equal volume of acetonitrile and centrifuged at $3000 \mathrm{~g}$ for 15 minutes. The supernatants were collected and 4-oxo-atRA formation was analyzed by LC-MS/MS as described above. All incubations were normalized to a no cofactor control.

Data Analysis. Mass spectrometry data were analyzed using Analyst software. Peak heights were used to determine the ratio of substrate to the internal standard. The areas under the medium and cell concentration versus time curves (AUCs) from 0 to 12 hours of $a t \mathrm{RA},(4 R)-\mathrm{OH}-a t \mathrm{RA},(4 S)-\mathrm{OH}-a t \mathrm{RA}, 4-0 \times 0-a t \mathrm{RA}$, and 18-OH-atRA were calculated by noncompartmental analysis using Phoenix/ WinNonLin 6.3 software (Pharsight, Mountain View, CA). The cellto-medium ratio was calculated for each inducer and for their subsequent metabolites using their respective $\mathrm{AUC}^{0-12} \mathrm{~h}$ in HepG2 cells and the media. One-way analyses of variance coupled with Bonferroni multiple comparison tests were conducted using GraphPad Prism software (version 5) to determine significant differences in mRNA expression. Significant changes in 4-oxo-atRA formation from atRA or 4-OH-atRA in the presence of inhibitors compared with no inhibitor controls were compared using unpaired $t$ test. $P<0.05$ was considered significant. The $K_{\mathrm{m}}$ values for 4-oxo-atRA and 18-OH-atRA were determined from the depletion data as previously described (Shimshoni et al., 2012)

\section{Results}

Induction of CYP26A1 and RAR $\beta$ in HepG2 Cells and Human Hepatocytes, and RAR Activation by $a t R A$ and Its Metabolites. The mRNA of CYP26A1 and RAR $\beta$ was significantly $(P<0.05)$ increased after 48 -hour treatment of

\section{TABLE 1}

Effect of atRA, 4-OH-atRA, and 4-oxo-atRA on $\operatorname{RAR} \alpha, \operatorname{RAR} \beta$, and RAR $\gamma$ activation in reporter assays

Data are presented as $\mathrm{EC}_{50}$ values with $95 \%$ confidence intervals.

\begin{tabular}{lccc}
\hline \multicolumn{1}{c}{ Treatment } & $\operatorname{RAR} \alpha$ & $\mathrm{RAR} \beta$ & $\mathrm{RAR} \gamma$ \\
\hline & & $n M$ & \\
atRA & $19(13-30)$ & $1(0.7-1.5)$ & $4(3-6)$ \\
4-OH- $a t$ RA & $114(91-141)$ & $53(33-84)$ & $45(37-54)$ \\
4-Oxo-atRA & $61(47-80)$ & $9(5-14)$ & $15(11-20)$ \\
\hline
\end{tabular}


Retinoid Concentrations:Media Fraction

A

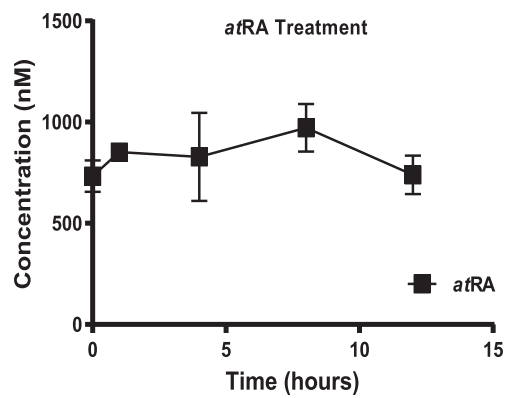

B

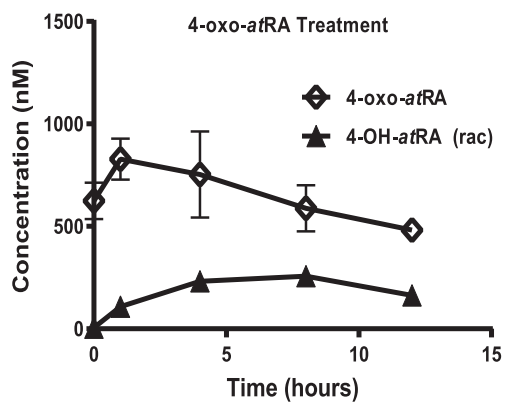

C

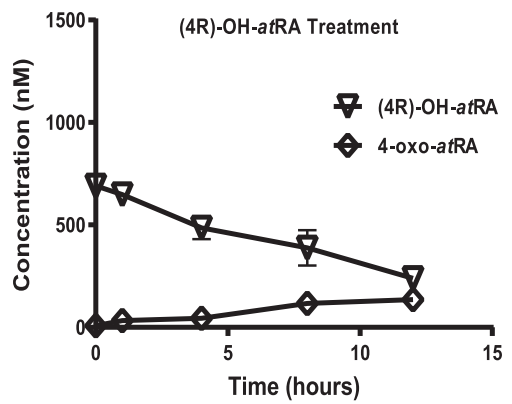

D

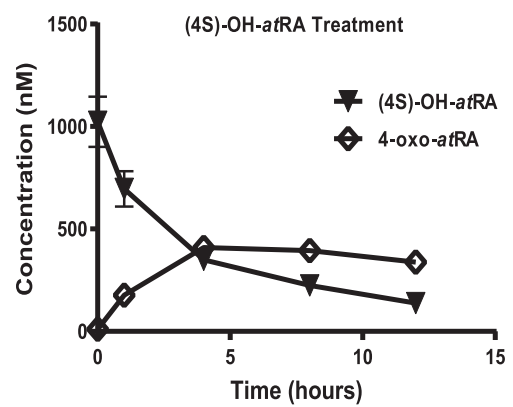

E

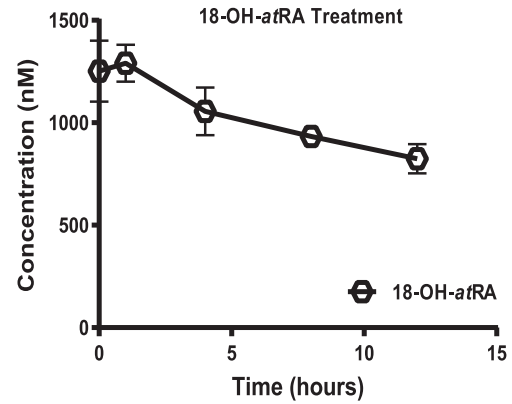

\section{Retinoid Concentrations:HepG2 Cellular Fraction}

$F$

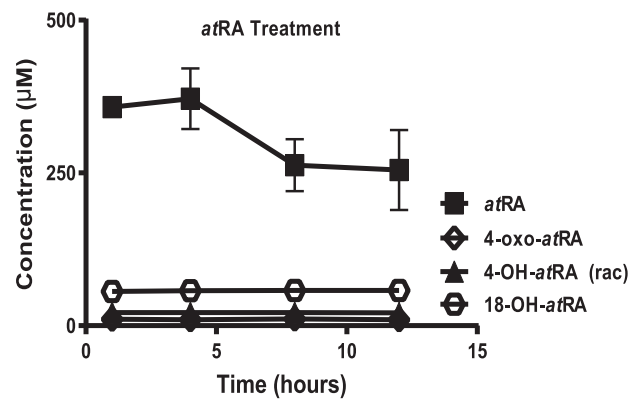

G

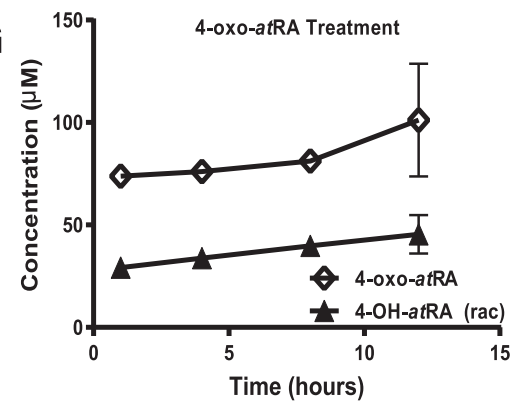

H

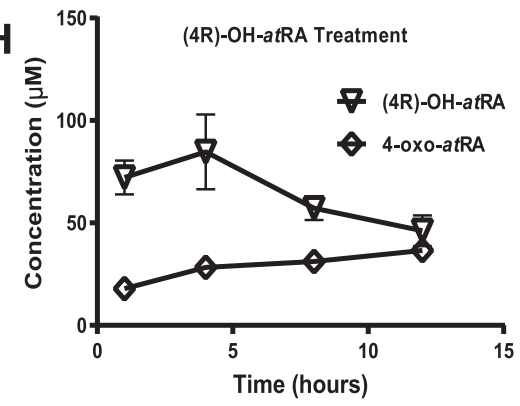

I

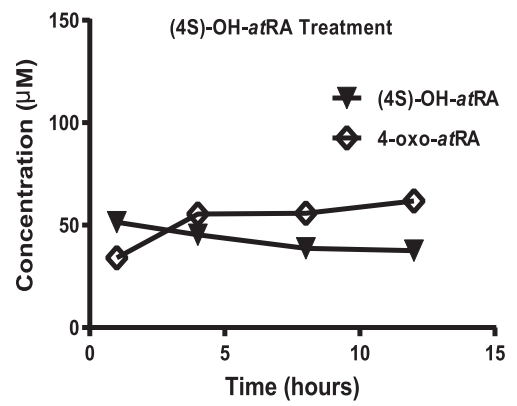

J

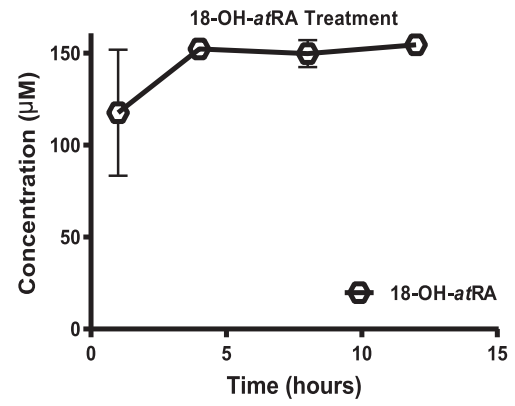

Fig. 5. Retinoids and their metabolite concentration versus time profiles in cell medium (A-E) and in the HepG2 cells (F-J) during a 12-hour treatment. (A) and (F) show the detected concentrations after treatment with atRA. (B) and (G) show the concentrations of detected retinoids after treatment with 4-oxo-atRA. $(\mathrm{C})$ and $(\mathrm{H})$ show detected retinoids after treatment with (4R)-OH-atRA. (D) and (I) show detected retinoids after treatment with $(4 S)-\mathrm{OH}-a t \mathrm{RA} .(\mathrm{E})$ and $(\mathrm{J})$ show the detected concentrations of 18-OH-RA after treatment with this retinoid. HepG2 cells were treated with each inducer $(1 \mu \mathrm{M})$ and aliquots were taken from the media (A-E) at $0,1,4,8$, and 12 hours. Cells (F-J) were collected for analysis at $1,4,8$, and 12 hours.
HepG2 cells with atRA, 4-oxo-atRA, (4R)-OH-atRA, (4S)-OH-atRA, and 18-OH-atRA $(1 \mu \mathrm{M})$, with atRA resulting in the greatest induction (Fig. 2). The induction of CYP26A1 and RAR $\beta$ was retinoid concentration dependent (Fig. 2, B and C). The magnitude of CYP26A1 induction after 4-oxo-atRA treatment (2980-fold,
$1 \mu \mathrm{M}$ treatment) was approximately $80 \%$ of that after atRA treatment (3700-fold, $1 \mu \mathrm{M}$ treatment), whereas the induction of CYP26A1 after 4-OH-atRA (both enantiomers) and 18-OH-atRA treatment was much less, 9-36\% of that observed after atRA treatment (Fig. 2A). The magnitude of induction of CYP26A1 
mRNA between (4R)-OH-atRA and (4S)-OH-atRA treatments was similar $(P>0.05)$. atRA and its metabolites also induced $\operatorname{RAR} \beta$ mRNA in a concentration-dependent manner, but the magnitude of $\operatorname{RAR} \beta$ induction was much less, approximately $3-18 \%$ of that observed with CYP26A1 (Fig. 2). There were no significant changes in $\mathrm{RAR} \alpha$ or $\operatorname{RAR} \gamma$ mRNA upon treatment with atRA or any of the atRA metabolites (data not shown).

Similar to HepG2 cells, atRA, 4-oxo-atRA, and 4-OH-atRA significantly increased CYP26A1 and $\operatorname{RAR} \beta$ mRNA in human hepatocytes (Fig. 3) and the magnitude of CYP26A1 induction was approximately 5 -fold greater than $\mathrm{RAR} \beta$ induction. In human hepatocytes, there was no significant induction of CRABP-I or CRABP-II by any of the three compounds. After the 2-hour treatment of human hepatocytes, at $\mathrm{RA}$ resulted in the greatest induction of CYP26A1 (16-fold, $1 \mu \mathrm{M}$ treatment), whereas 4-OH-atRA was a weaker inducer (12-fold induction). The magnitude of CYP26A1 induction was substantially less in human hepatocytes after 2-hour treatment than in HepG2 cells after a 48-hour treatment.

In reporter cell lines, atRA was the most potent activator of all three RARs. 4-Oxo-atRA was 3- to 8-fold less potent than atRA (Fig. 4; Table 1). 4-OH-atRA was the least potent activator of all three RARs. It was 2 - to 6 -fold less potent than 4-oxo-atRA and 6- to 50-fold less potent than atRA in activating the three RARs (Table 1).

Retinoid Concentrations and Exposure in HepG2 Cells and Hepatocytes. To determine whether the specific metabolites used in the HepG2 cell treatments were responsible for CYP26A1 and RAR $\beta$ induction, retinoid concentrations and $\mathrm{AUC}^{0-12 \mathrm{~h}}$ both in the medium and in cells were quantified after treatment with atRA or the metabolites (Fig. 5; Table 2). The initial concentration of each treatment was $1 \mu \mathrm{M}$; however, because of significant depletion of the inducers during incubations, the average concentration in cells during treatment was within physiologic concentrations of these compounds. In terms of absolute concentrations, at RA had the highest cell $\mathrm{AUC}^{0-12} \mathrm{~h}(3750 \pm 350 \mathrm{nmol} \cdot \mathrm{h} / \mathrm{ml})$, whereas (4S)-OH-atRA had the lowest $\mathrm{AUC}^{0-12 \mathrm{~h}}(300 \pm 10 \mathrm{nmol} \cdot \mathrm{h} / \mathrm{ml})$ (Table 2). Although exposures to the 4-OH-atRA enantiomers were $90 \%$ lower than to atRA, the magnitude of CYP26A1 induction after treatment with 4-OH-atRA enantiomers was nearly $40 \%$ of that observed after atRA treatment, suggesting that 4-OH-atRA enantiomers or their metabolites are more potent activators of RAR than atRA. The cell $\mathrm{AUC}^{0-12 \mathrm{~h}}$ of 4-oxo-atRA $(980 \pm 40 \mathrm{nmol} \cdot \mathrm{h} / \mathrm{ml})$ was $60 \%$ lower than that of atRA, but treatment with 4-oxo-atRA resulted in a similar induction of CYP26A1 and $\operatorname{RAR} \beta$ as atRA, suggesting that 4-oxo-atRA is a more potent inducer of CYP26A1 and RAR $\beta$ than atRA (Table 2). The cell-to-medium concentration ratio ranged from 70 for ( $4 S$ )-OH-atRA to 360 for atRA, demonstrating differential partitioning of these retinoids into cells (Table 2).

Depletion of atRA was not observed during the first 12 hours of treatment (Figs. 5 and 6) and no formation of 4-OH-atRA or 4-oxo-atRA was detected in the medium during this time. In the cells, 4-OH-atRA and 4-oxo-atRA were detected at concentrations of approximately 3\% of atRA after 12 hours of atRA treatment, suggesting minimal CYP26 activity at baseline in the HepG2 cells. Similar to atRA depletion, minimal depletion (up to $30 \%$ ) of 4-oxo-atRA and 18-OH-atRA was observed in medium and cells during the first 12 hours of treatment (Figs. 5 and 6). Depletion of the 4-OH-atRA enantiomers was much more

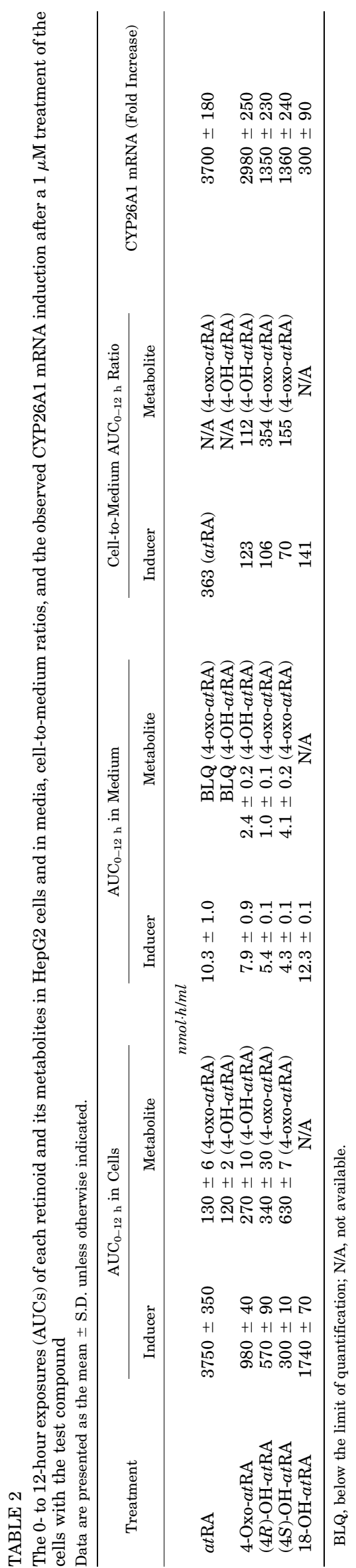


rapid than observed for atRA, 4-oxo-atRA, or 18-OH-atRA. During the first 12 hours of treatment $86 \%$ of $(4 S)-\mathrm{OH}-a t \mathrm{RA}$ and $65 \%$ of ( $4 R$ )-OH-atRA were depleted (Figs. 5 and 6 ). This depletion was accompanied by increased 4-oxo-at RA concentrations in the cells and medium, and the concentrations of 4-oxo-atRA in the cells were similar to those of 4-OH-atRA after treatments with the 4-OH-atRA enantiomers (Fig. 5).

After the first 24 hours of treatment, increased depletion of at RA (61\%) was detected (Fig. 6) and 4-OH-atRA, 4-oxo-atRA, and 18-OH-atRA were detected as metabolites of atRA in both the cells and the medium. The depletion of 18-OH-at RA and 4-oxo-atRA was increased after 24 and 48 hours of treatment; $62 \%$ of $18-\mathrm{OH}-a t \mathrm{RA}$ and $29 \%$ of 4 -oxo-at RA were depleted at 24 hours and over $94 \%$ of both compounds were depleted at 48 hours (Fig. 6). (4R)-OH-atRA depletion was $89 \%$ and (4S)-OH-atRA depletion was $87 \%$ after 24 hours of treatment (Fig. 6).

In human hepatocytes, depletion of atRA, 4-OH-atRA, and 4-oxo-atRA, and formation of metabolites was observed already at 2 hours of treatment (Table 3 ). The medium exposure $\left(\mathrm{AUC}^{0-2} \mathrm{~h}\right)$ for 4-oxo-atRA was approximately $50 \%$ lower than that of atRA at all treatment concentrations, whereas the exposure to 4-OH-atRA was similar to that of atRA.

Time Course of CYP26A1 and RAR $\beta$ Induction in HepG2 Cells. To determine whether the time course of depletion for atRA, 4-oxo-atRA, and 18-OH-atRA could be explained by induction of CYP26A1 activity, the time course of CYP26A1 activity and CYP26A1 and $\operatorname{RAR} \beta$ mRNA was quantified. The time course of CYP26A1 mRNA induction in HepG2 cells is shown in Fig. 7 together with the time course of $\mathrm{RAR} \beta$ and CRABP-II induction. CYP26A1 activity was measured by quantifying the formation of 4-oxo-atRA- $d_{3}$ and 4-OH- $a t \mathrm{RA}-d_{4}$ from $a t \mathrm{RA}-d_{5}$ at designated time points over a 48-hour treatment with atRA $(1 \mu \mathrm{M})$. Formation of 4-OH-at RA- $d_{4}$ was slow but detectable during the first 12 hours after initial treatment with atRA. It then increased approximately 7 -fold $(P<0.05)$ at 24 hours and 33 -fold $(P<0.05)$ at 48 hours after the initial treatment with atRA (Fig. 7). Formation of 4-oxo- $a t$ RA- $d_{3}$ increased similarly as 4-OH-atRA- $d_{4}$, approximately 7 -fold $(P<0.05)$ at 24 hours and 22 -fold $(P<0.05)$ at 48 hours after the treatment with $a t \mathrm{RA}$ (Fig. 7C). The ratio of 4-OH- $a t \mathrm{RA}-d_{4}$ to 4 -oxo- $a t \mathrm{RA}-d_{3}$ did not change with time. The time course of CYP26A1 activity was in agreement with atRA and 18-OH-atRA depletion but not with 4-OH-at RA depletion.

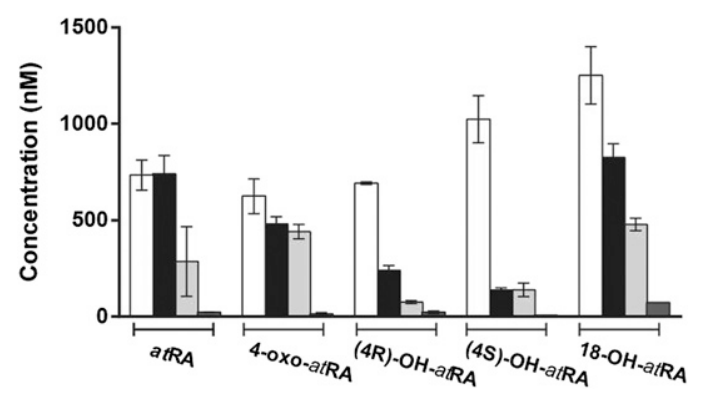

Fig. 6. Metabolism of the retinoids after $a t \mathrm{RA}$ and metabolite treatment. atRA, $(4 R)-\mathrm{OH}-a t \mathrm{RA},(4 S)-\mathrm{OH}-a t \mathrm{RA}, 4-0 \times 0-a t \mathrm{RA}$, and 18-OH-atRA concentrations at 0 (white bars), 12 (black bars), 24 (light gray bars), and 48 (dark gray bars) hours after $1 \mu \mathrm{M}$ treatment with each of the compounds in HepG2 cells. Medium containing the inducer was changed at 24 hours after a 1-ml aliquot was taken for analysis.
TABLE 3

The 0- to 2-hour exposures (AUCs) of each retinoid and its metabolites in human primary hepatocytes

Data are presented as the mean \pm S.D. unless otherwise indicated.

\begin{tabular}{|c|c|c|}
\hline \multirow{2}{*}{ Treatment } & \multicolumn{2}{|c|}{$\mathrm{AUC}_{0-12 \mathrm{~h}}$ in Medium } \\
\hline & Inducer & Metabolites \\
\hline & \multicolumn{2}{|c|}{$\mathrm{nmol} \cdot \mathrm{h} / \mathrm{ml}$} \\
\hline \multicolumn{3}{|l|}{ atRA } \\
\hline $100 \mathrm{nM}$ & $302 \pm 7$ & BLQ \\
\hline $500 \mathrm{nM}$ & $1305 \pm 16$ & BLQ \\
\hline $1 \mu \mathrm{M}$ & $2810 \pm 62$ & $0.6 \pm 0.4(4$-oxo- $a t \mathrm{RA})$ \\
\hline \multicolumn{3}{|l|}{ 4-OH-atRA } \\
\hline $100 \mathrm{nM}$ & $277 \pm 5$ & $11 \pm 2(4-$ oxo- $a t \mathrm{RA})$ \\
\hline $500 \mathrm{nM}$ & $848 \pm 6$ & $90 \pm 5$ (4-oxo-atRA) \\
\hline $1 \mu \mathrm{M}$ & $2018 \pm 12$ & $309 \pm 20$ (4-oxo-atRA) \\
\hline \multicolumn{3}{|l|}{ 4-Oxo-atRA } \\
\hline $100 \mathrm{nM}$ & $172 \pm 3$ & $0(4-\mathrm{OH}-a t \mathrm{RA})$ \\
\hline $500 \mathrm{nM}$ & $728 \pm 10$ & $6.5 \pm 0.5(4-\mathrm{OH}-a t \mathrm{RA})$ \\
\hline $1 \mu \mathrm{M}$ & $1584 \pm 29$ & $11 \pm 2(4-\mathrm{OH}-a t \mathrm{RA})$ \\
\hline
\end{tabular}

$\mathrm{BLQ}$, below the limit of quantification.

Formation of 4-Oxo-atRA from 4-OH-atRA in HepG2 Cells, Human Hepatocytes, and Human Liver Microsomes. The major metabolite formed from both 4-OH-atRA enantiomers was 4-oxo-atRA and the metabolism of 4-OH-atRA enantiomers was stereoselective. The cell and medium AUCs for $(4 S)-\mathrm{OH}-a t \mathrm{RA}$ were lower than those for $(4 R)-\mathrm{OH}-a t \mathrm{RA}$, suggesting a greater clearance of $(4 S)-\mathrm{OH}-a t \mathrm{RA}$ in HepG2 cells. The AUC ${ }^{0-12 \mathrm{~h}}$ of 4-oxo-atRA as a metabolite of (4S)-OH-atRA was 2.1-fold higher than (4S)-OH-at RA cell AUC ${ }^{0-12 \mathrm{~h}}$ (Table 2, metabolite-to-parent ratio 2.1), whereas the $\mathrm{AUC}^{0-12 \mathrm{~h}}$ of 4-oxo-atRA was $40 \%$ lower than ( $4 R$ )-OH-atRA in cells treated with (4R)-OH-RA (Table 2, metabolite-to-parent ratio 0.6). Consistent with the HepG2 cell metabolite-to-parent ratios, 4-oxo-at RA AUC ${ }^{0-12 \mathrm{~h}}$ was $25 \%$ lower than (4R)-OH-at RA AUC ${ }^{0-12 \mathrm{~h}}$ in the medium, whereas 4-oxo-atRA and (4S)-OH-atRA had equal AUCs. On the basis of these data, after (4S)-OH-atRA treatment, 4-oxo-atRA is the predominant retinoid in the cells, and the formation clearance of 4-oxo-atRA from (4S)-OH-atRA is higher than the formation clearance of 4-oxo-at $\mathrm{RA}$ from $(4 R)$ $\mathrm{OH}-a t \mathrm{RA}$. Interestingly, 4-OH-atRA was also detected as a reductive metabolite in HepG2 cells treated with 4-oxo-atRA and had an $\mathrm{AUC}^{0-12 \mathrm{~h}}$ that was $40 \%$ of that of 4-oxo-at RA in cells (Fig. 4; Table 2). No degradation of 4-oxo-atRA, (4R)-OH-atRA, or (4S)-OH-atRA was observed in the medium in the absence of cells (data not shown), indicating that the reversible oxidation/ reduction seen in HepG2 cells is cell mediated.

In human hepatocytes treated with atRA for 2 hours, only 4oxo-atRA was detected as a metabolite; in 4-OH-atRA-treated hepatocytes, significant formation of 4-oxo-at RA was observed at 2 hours of treatment. 4-Oxo-atRA concentrations in the media were $5-10 \%$ of those of $4-\mathrm{OH}$-atRA after $4-\mathrm{OH}-a t \mathrm{RA}$ treatment (Table 3). Formation of 4-OH-atRA was also observed in the 4-oxo-atRA-treated cells (Table 3).

To determine whether formation of 4-oxo-at RA from 4-OH-at RA enantiomers is P450 mediated, HepG2 cells were first coincubated with ketoconazole and atRA or racemic 4-OH-atRA. Because formation of 4-OH-RA from atRA is predominantly CYP26A1 mediated, inhibition of 4-OH-atRA formation from atRA by ketoconazole was used as a positive control for CYP26A1 inhibition. In cells treated with atRA, ketoconazole decreased 4-OH-atRA formation by $84 \%(P<0.05)$ and subsequent 4-oxoatRA formation by $80 \%(P<0.05)$ compared with the control 

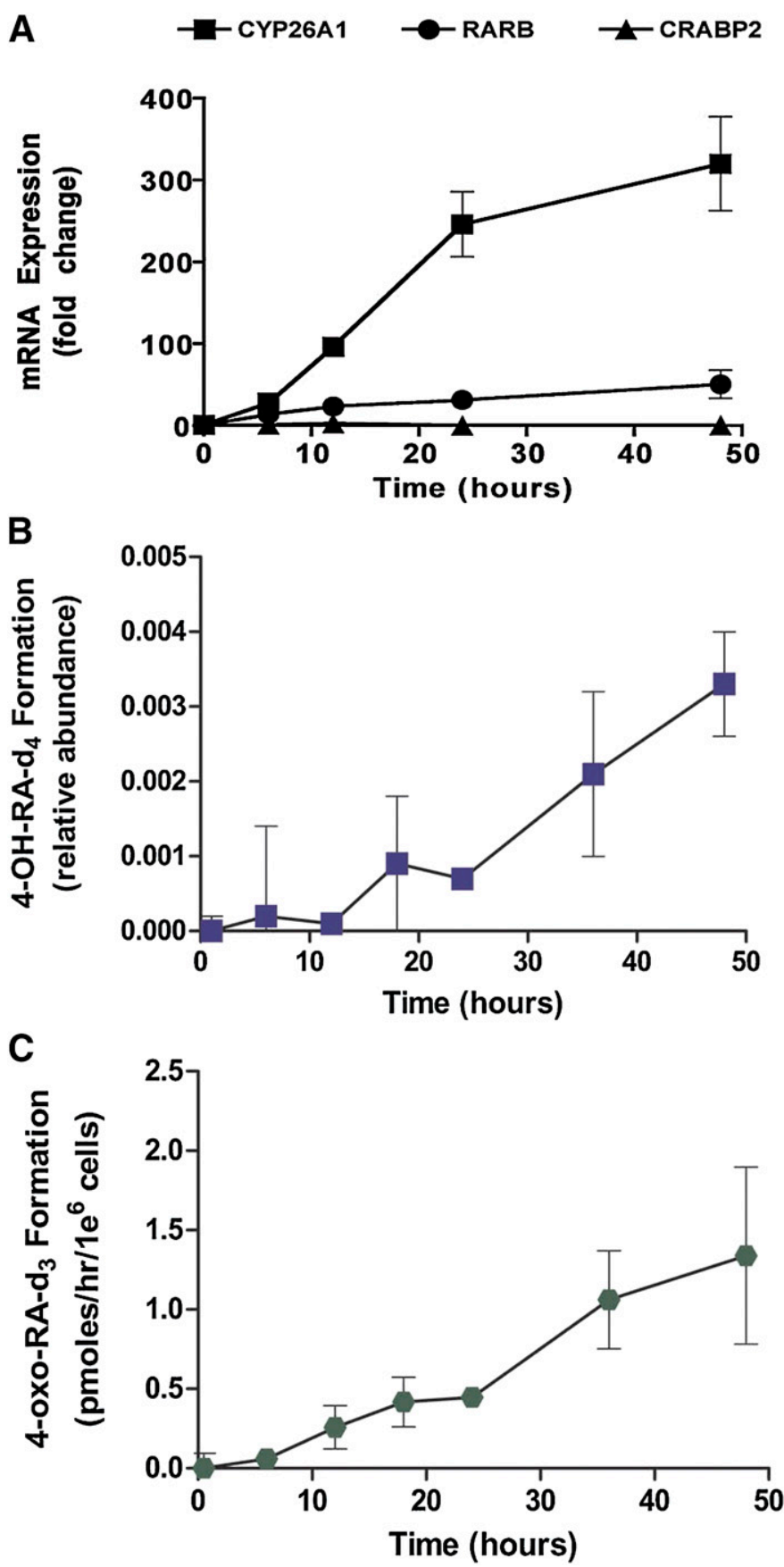

Fig. 7. Time course of CYP26A1 mRNA induction and activity in the HepG2 cells after atRA and metabolite treatments. (A) Time course of CYP26A1, RAR $\beta$, and CRABP-I mRNA induction in HepG2 cells after treatment with $1 \mu \mathrm{M}$ atRA. (B and C) Time course of atRA- $d_{5}$ metabolism during induction with atRA. The formation of 4-OH-RA- $d_{4}(\mathrm{~B})$ and 4-oxo$a t$ RA- $d_{3}$ (C) after 1-hour treatments of $a t \mathrm{RA}-d_{5}(100 \mathrm{nM})$ is shown at selected time points (ranging from 0.5 to 48 hours) during the 48-hour treatment with atRA $(1 \mu \mathrm{M})$.

cells (Table 4). Yet ketoconazole had no effect on 4-oxo-atRA formation in cells treated with 4-OH-atRA. The depletion of 4$\mathrm{OH}-a t \mathrm{RA}$ was decreased by $25 \%(P<0.05)$ by ketoconazole in agreement with a fraction of 4-OH-atRA cleared by CYP26A1 to products other than 4-oxo-atRA. When HepG2 cells were cotreated with atRA and talarozole (a CYP26-specific inhibitor), 4-OH-RA and 4-oxo-RA formation was significantly decreased, confirming that 4-OH-atRA formation in these cells is predominantly CYP26A1 mediated (Table 4). None of the other inhibitors tested decreased 4-oxo-atRA formation from 4-OH-atRA or atRA (Table 4).

To further evaluate the enzymes responsible for 4-oxo-atRA formation from 4-OH-atRA in the human liver, human liver microsomes were incubated with 4-OH-at RA in the presence of three different cofactors: $\mathrm{NADPH}, \mathrm{NADP}^{+}$, and $\mathrm{NAD}^{+}$. All three cofactors supported 4-oxo-atRA formation (Fig. 8), but formation of 4-oxo-atRA in the presence of $\mathrm{NAD}^{+}$was up to 4 -fold higher than in the presence of NADPH or $\mathrm{NADP}^{+}$. Talarozole (a preferential CYP26A1 inhibitor) did not inhibit the NADPH-mediated formation of 4-oxo-atRA from 4-OH-atRA, whereas ketoconazole (a pan-P450 inhibitor) decreased the NADPH-mediated formation of 4-oxo-atRA by $50 \%$.

Metabolism of 4-Oxo-atRA and 18-OH-atRA by CYP26A1. The depletion of 4-oxo-atRA and 18-OH-atRA in HepG2 cells was observed after increased CYP26A1 activity, suggesting that both 18-OH-atRA and 4-oxo-at RA were cleared by CYP26A1. To determine the role of CYP26A1 in clearing 18-OH-atRA and 4-oxo-atRA, the depletion kinetics of both compounds was determined using recombinant CYP26A1 (Fig. 9). Both 18-OH-atRA and 4-oxo-atRA were efficiently depleted by CYP26A1. The $K_{\mathrm{m}}$ values for 4-oxo-atRA and 18-OHatRA depletion by CYP26A1 were 63.0 and $38.5 \mathrm{nM}$, respectively. Intrinsic clearance $\left(\mathrm{CL}_{\text {int }}\right)$ by CYP26A1 was 2.5 -fold higher for 18-OH-atRA $(230 \pm 43 \mu \mathrm{l} / \mathrm{min}$ per pmol P450) than for 4-oxoat RA $(90 \pm 9.0 \mu \mathrm{l} / \mathrm{min}$ per pmol $\mathrm{P} 450)$.

\section{Discussion}

This study shows that metabolites of atRA induce RAR $\beta$ and CYP26A1 mRNA but only 4-oxo-atRA is an equipotent or more potent inducer than atRA. The induction of CYP26A1 mRNA observed in the HepG2 cells after atRA treatment is consistent with previous studies of CYP26A1 induction by atRA (Tay et al., 2010; Zhang et al., 2010). Similarly, the lower magnitude of RAR $\beta$ induction compared with CYP26A1 is in agreement with previous results (Chambon, 1996; Tay et al., 2010). The fold induction of CYP26A1 was higher in HepG2 cells than in human hepatocytes, but overall the results in human hepatocytes supported the findings in HepG2 cells. The lower fold induction in hepatocytes may be due to higher baseline expression of CYP26A1 in hepatocytes compared with HepG2 cells or the much shorter induction time ( 2 hours) in hepatocytes than in HepG2 cells (48 hours). As shown in Fig. 6, the mRNA induction peaks at 48 hours in HepG2 cells. However, the short incubation time was chosen for hepatocytes to minimize metabolism and depletion of the inducers.

Induction of CYP26A1 and RAR $\beta$ mRNA in HepG2 cells was previously shown to be RAR $\alpha$ mediated (Tay et al., 2010), and the potency of CYP26A1 induction by the metabolites of atRA is expected to follow the binding affinity of the metabolites to $\operatorname{RAR} \alpha$. Based on previous studies in transfected COS-7 cells, which are expected to have minimal CYP26A1 activity, 4-oxo-atRA is the most potent activator of $\operatorname{RAR} \alpha\left(\mathrm{EC}_{50}=33 \mathrm{nM}\right)$, with at RA and 18-OH-RA being 5 -fold less potent $\left(\mathrm{EC}_{50}=169\right.$ and $162 \mathrm{nM}$, respectively) (Idres et al., 2002). However, in the current study in the reporter assays, atRA was about 3-fold more potent $\operatorname{RAR} \alpha$ agonist and 8-fold more potent $\operatorname{RAR} \beta$ agonist than was 4-oxo-atRA. Yet, in human hepatocytes atRA and 4-oxo-atRA had similar potency as CYP26 inducers. In HepG2 cells 18-OH-atRA was significantly 
TABLE 4

The effect of selective P450 and alcohol dehydrogenase inhibitors on 4oxo-at RA or 4-OH-atRA formation from atRA and formation of 4-oxo-at $\mathrm{RA}$ from 4-OH-atRA in HepG2 cells

Data are presented as the percentage of control (mean \pm S.D.) unless otherwise indicated. The inhibitors used were 4-methylpyrazole $(250 \mu \mathrm{M}$, alcohol dehydrogenases), cimetidine ( $250 \mu \mathrm{M}$, alcohol dehydrogenases), ketoconazole (10 $\mu \mathrm{M}, \mathrm{P} 450 \mathrm{~s})$, talarozole $(1 \mu \mathrm{M}$, CYP26), testosterone $(25 \mu \mathrm{M}$, aldo-keto reductases), or carbenoxolone (25 and $50 \mu \mathrm{M}$, retinol dehydrogenases). Significant changes in comparison with no inhibitor controls were performed using the unpaired $t$ test.

\begin{tabular}{lcc}
\hline \multicolumn{1}{c}{ Treatment } & $\begin{array}{c}\text { 4-oxo-atRA } \\
\text { Formation }\end{array}$ & $\begin{array}{c}\text { 4-OH-atRA } \\
\text { Concentration }\end{array}$ \\
\hline atRA (control) & $100 \pm 3$ & $100 \pm 7$ \\
atRA + 4-methylpyrazole & $109 \pm 8$ & $122 \pm 11$ \\
atRA+ cimetidine & $130 \pm 6^{*}$ & $149 \pm 5^{*}$ \\
atRA+ ketoconazole $(10 \mu \mathrm{M})$ & $20 \pm 1^{*}$ & $16 \pm 6^{*}$ \\
atRA+ testosterone & $109 \pm 6$ & $133 \pm 4^{*}$ \\
atRA + carbenoxolone $(25 \mu \mathrm{M})$ & $74 \pm 3^{*}$ & $92 \pm 22$ \\
atRA + carbenoxolone $(50 \mu \mathrm{M})$ & $80 \pm 8^{*}$ & $99 \pm 25$ \\
atRA + talarozole $(1 \mu \mathrm{M})$ & $20 \pm 3^{*}$ & 0 \\
4-OH-atRA (control) & $100 \pm 6$ & $100 \pm 6$ \\
4-OH-atRA + 4- & $121 \pm 12$ & $96 \pm 3$ \\
$\quad$ methylpyrazole & $121 \pm 3^{*}$ & $99 \pm 2$ \\
4-OH-atRA + cimetidine & $110 \pm 0$ & $75 \pm 0^{*}$ \\
4-OH-atRA + ketoconazole & $107 \pm 1$ & $107 \pm 0$ \\
4-OH-atRA + testosterone & $115 \pm 9$ & $80 \pm 9^{*}$ \\
4-OH-atRA + carbenoxolone & & \\
$\quad(25 \mu \mathrm{M})$ & $117 \pm 6^{*}$ & $96 \pm 3$ \\
4-OH-atRA + carbenoxolone & & \\
$\quad(50 \mu \mathrm{M})$ &
\end{tabular}

$* P<0.05$.

less potent than atRA or 4-oxo-atRA in inducing CYP26A1 and $\operatorname{RAR} \beta$, while 4-oxo-atRA and atRA were equipotent. These differences between studies and cell lines could be due to differential partitioning of the compounds into cells or expression of CRABPs. CRABPs may affect RAR activation and bind atRA with higher affinity than the metabolites. However, no CRABP-II induction was observed in HepG2 cells and CRABP-I was not expressed in HepG2 cells, suggesting that changes in binding protein expression do not contribute to observed induction.

In transfected COS-7 cells, 4-OH-at RA was the least potent activator of $\operatorname{RAR} \alpha\left(\mathrm{EC}_{50}=791 \mathrm{nM}\right)$ (Idres et al., 2002). Similarly, in our studies, 4-OH-atRA was the least potent RAR agonist for all three RAR isoforms. However, in our studies in HepG2 cells, the CYP26A1 induction after 4-OHatRA treatment was approximately one-third of that observed with atRA; in human hepatocytes, the induction of CYP26A1 by $4-\mathrm{OH}$-atRA was about $30 \%$ less than that observed after $1 \mu \mathrm{M}$ atRA treatment. This difference between the reporter cell systems and HepG2 cells or hepatocytes is likely due to differences in formation of 4-oxo-atRA, because all of the data presented here support the interpretation that induction of RAR target genes after 4-OH-atRA treatments is largely attributable to the formation of 4-oxo-at RA.

Previous studies have shown that atRA, 4-OH-atRA, 18OH-atRA, and 4-oxo-atRA are substrates of CYP26A1, and CYP26A1 forms a variety of diols and oxo-alcohols from these metabolites (Lutz et al., 2009; Shimshoni et al., 2012; Topletz et al., 2012). Although synthetic standards are not available for these sequential metabolites, the depletion clearance of the metabolites can be used to evaluate the role of CYP26A1 in their clearance. Using recombinant CYP26A1, atRA and (4S)-OH-at RA were found to have the highest $\mathrm{CL}_{\mathrm{int}}(1.2-$ $1.4 \mathrm{ml} / \mathrm{min}$ per pmol) and 4-oxo-atRA the lowest $\mathrm{CL}_{\text {int }}(0.09 \mathrm{ml} / \mathrm{min}$ per pmol) by CYP26A1. Interestingly, in HepG2 cells, 4-OHatRA had more than 2-fold higher clearance than atRA, and the clearance of atRA was of similar magnitude as 4-oxo-atRA. The high clearance of 4-OH-atRA and 4-oxo-at RA compared with atRA in HepG2 cells suggests that multiple enzymes are involved in their elimination, and that the induction state of CYP26A1 affects the relative importance of CYP26A1 in retinoid clearance. It is well established that CYP26A1 is the main enzyme that clears atRA, and ketoconazole and talarozole effectively inhibited the metabolism of atRA in HepG2 cells, confirming the role of CYP26A1. Incubations with labeled atRA showed that there is minimal baseline CYP26A1 activity in HepG2 cells, a finding in agreement with the induction time course of CYP26A1 mRNA. However, on the basis of the data presented, there are additional enzymes already at baseline in the cells that clear 4-oxo-atRA and 4-OH-atRA. It is possible that glucuronidation of 4-OH-atRA (and the other retinoids via acyl glucuronides) contributes to 4-OH-atRA clearance in HepG2 cells and in hepatocytes, because UGT2B7 has been shown to glucuronidate 4-OH-atRA (Samokyszyn et al., 2000) and glucuronides are common in vivo metabolites of atRA. In addition, other additional enzymatic processes in the clearance of 4-OH-atRA and 4-oxo-atRA are supported by the observation of the reduction of 4-oxo-at RA back to 4-OH-atRA in the treated HepG2 cells and human hepatocytes, a reaction that is not likely to be P450 mediated. However, CYP26A1 appears to contribute to the clearance of 4-oxo-atRA and 4-OH-atRA because the clearance of these compounds was significantly greater after 48 hours of atRA treatment than during the first 24 hours.

Despite the fact that all metabolites are substrates of CYP26A1 and their clearances increase with increasing CYP26A1 activity, formation of 4-oxo-atRA from 4-OHat RA appears not to be CYP26A1 mediated in HepG2 cells

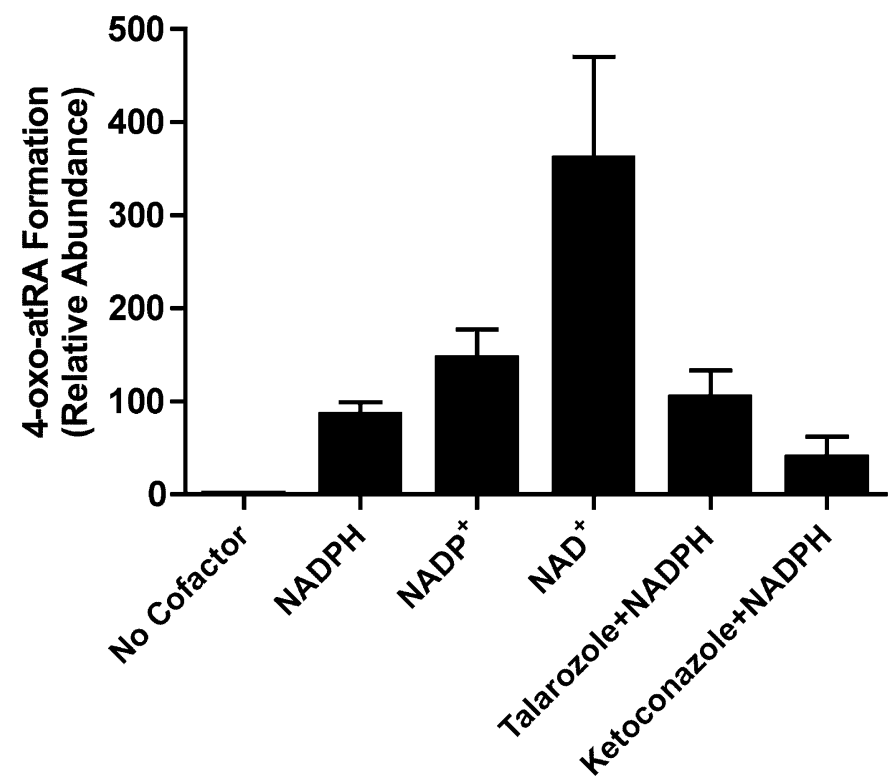

Fig. 8. Cofactor dependence in the formation of 4-oxo-atRA from 4-OHatRA in human liver microsomes. Human liver microsomes were incubated with $500 \mathrm{nM}$ 4-OH-atRA with $\mathrm{NADPH}, \mathrm{NAD}^{+}$, or $\mathrm{NADP}^{+}$as a cofactor and 4-oxo-atRA formation measured. In addition, the inhibition of 4-oxo-atRA formation from 4-OH-atRA in the presence of NADPH by ketoconazole $(10 \mu \mathrm{M})$ and talarozole $(1 \mu \mathrm{M})$ was measured. 

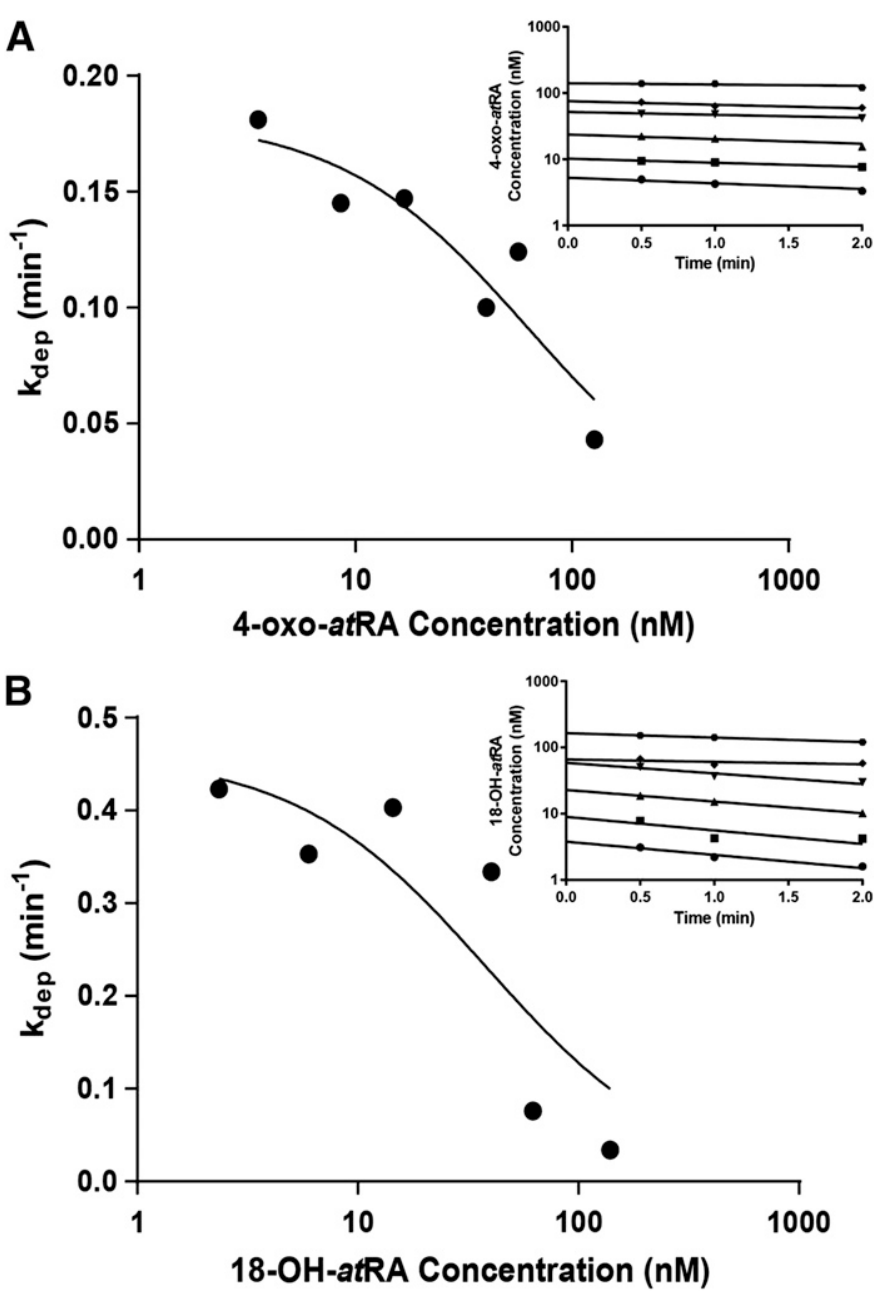

Fig. 9. Metabolism of 4-oxo-atRA and 18-OH-atRA by CYP26A1. The depletion of 4-oxo-at RA (A) and 18-OH-atRA (B) as a function of time was measured at six different concentrations of each substrate and the depletion rates were determined (insets in both panels). All depletion experiments were conducted in duplicate. The depletion constants as a function of substrate concentrations were used to determine the $K_{\mathrm{m}}$ and $k_{\mathrm{dep} \text { max }}$ of 4-oxo-atRA and 18-OH-atRA with CYP26A1.

or in human liver. In HepG2 cells, there was no change in 4-oxo-at RA formation from 4-OH-at RA in the presence of the P450-inhibitor ketoconazole. Surprisingly, the formation of 4-oxo-atRA from 4-OH-atRA was also not decreased in the presence of inhibitors of several alcohol dehydrogenases, aldoketoreductases, or membrane-bound retinol dehydrogenases in HepG2 cells, suggesting that the enzyme responsible for 4-oxo-atRA formation is yet to be identified. In human liver microsomes, the formation of 4-oxo-atRA was supported by $\mathrm{NAD}^{+}$and to a lesser extent $\mathrm{NADP}^{+}$and $\mathrm{NADPH}$, suggesting that a microsomal alcohol dehydrogenase may be responsible for the majority of 4-oxo-at RA formation in human liver. These findings are in agreement with a previous report in which 4-oxo-atRA formation was found to be $\mathrm{NAD}^{+}$and $\mathrm{NADP}^{+}$mediated in hamster liver microsomes (Roberts et al., 1980). However, in contrast with the hamster liver microsomes, there are also P450 enzymes in the human liver that can carry out this reaction, as shown by the fact that NADPH could support the 4-oxo-atRA formation and this formation was inhibited partially by ketoconazole. Yet no inhibition of 4-oxo-atRA formation was observed with talarozole (a CYP26A1 inhibitor), showing that the formation of 4-oxo-atRA is not mediated by CYP26A1.

The formation of 4-oxo-atRA by a novel enzyme is noteworthy because 4-oxo-atRA is the only nonglucuronidated atRA metabolite detected in human plasma (Muindi et al., 1992a, 2008; Arnold et al., 2012). 4-Oxo-atRA accounts for $10-20 \%$ of the atRA dose in vivo, both in plasma and as 4-oxo-RAglucuronide in the urine (Muindi et al., 1992a, 2008; Arnold et al., 2012), making it a significant metabolite. The abundance of 4-oxo-atRA together with its proven biologic activity in animal models suggests that 4-oxo-atRA formation may contribute to some of the observed retinoid effects in vivo. The conclusive determination of the biologic importance of 4-oxo-atRA in vitamin A activity will require identification of the specific enzyme(s) that form this metabolite from 4-OH-atRA.

The findings presented showing that atRA and its metabolites induce CYP26A1 expression, and hence their own clearance, have implications in the broader context of understanding at RA resistance during therapy with at RA. The autoinduction of atRA clearance limits efficacy of atRA in various cancer treatments. The data presented here suggest that induction of CYP26A1 that results in increased metabolism of atRA and 4-oxo-atRA is a major contributor to atRA resistance. However, it has also been suggested that pregnane $\mathrm{X}$ receptor activation by comedications induces at $\mathrm{RA}$ clearance in the liver, likely via CYP3A4 induction (Wang et al., 2008). Further in vivo studies involving administration of the metabolites of atRA are needed to completely characterize the mechanisms involved in at RA resistance and the role that the metabolites may play.

In conclusion, this study shows that CYP26A1 functions primarily as an enzyme that metabolizes at RA, 4-OH-atRA, 18-OH-atRA, and 4-oxo-atRA into biologically inactive retinoids and does not contribute to the formation of metabolites with distinct retinoid activity. The results demonstrate that 4-oxo-atRA, (4S)-OH-atRA, (4R)-OH-atRA, and 18-OH-atRA induce CYP26A1 and therefore also their own formation. This autoinduction may have broader clinical significance because retinoid resistance is commonly believed to be a result of induction of CYP26A1 in target tissues and cells leading to resistance to therapeutic retinoids. The formation of the most potent inducer of RAR target genes (4-oxo-atRA from 4-OHatRA) likely depends on an unidentified microsomal alcohol dehydrogenase, suggesting that 4-oxo-atRA may have a biologic role independent of atRA. Further evaluation of the in vivo effects of 4-oxo-at RA and 4-OH-at RA is warranted to determine the full biologic significance of these metabolites and the enzymes forming them.

\section{Acknowledgments}

The authors thank Faith Stevison for skillful assistance with LC-MS/MS

\section{Authorship Contributions}

Participated in research design: Topletz, Tripathy, Foti, Nelson, Isoherranen.

Conducted experiments: Topletz, Tripathy, Foti.

Contributed new reagents or analytic tools: Topletz, Shimshoni, Nelson.

Performed data analysis: Topletz, Tripathy, Foti, Isoherranen.

Wrote or contributed to the writing of the manuscript: Topletz, Tripathy, Foti, Nelson, Isoherranen. 


\section{References}

Arnold SL, Amory JK, Walsh TJ, and Isoherranen N (2012) A sensitive and specific method for measurement of multiple retinoids in human serum with UHPLC-MS/ MS. J Lipid Res 53:587-598.

Baron JM, Heise R, Blaner WS, Neis M, Joussen S, Dreuw A, Marquardt Y, Saurat JH, Merk HF, and Bickers DR et al. (2005) Retinoic acid and its 4-oxo metabolites are functionally active in human skin cells in vitro. J Invest Dermatol 125 143-153.

Bonet ML, Ribot J, and Palou A (2012) Lipid metabolism in mammalian tissues and its control by retinoic acid. Biochim Biophys Acta 1821:177-189.

Chambon P (1996) A decade of molecular biology of retinoic acid receptors. FASEB J 10:940-954.

Chithalen JV, Luu L, Petkovich M, and Jones G (2002) HPLC-MS/MS analysis of the products generated from all-trans-retinoic acid using recombinant human CYP26A. J Lipid Res 43:1133-1142.

Chung SS and Wolgemuth DJ (2004) Role of retinoid signaling in the regulation of spermatogenesis. Cytogenet Genome Res 105:189-202.

Creech Kraft J, Löfberg B, Chahoud I, Bochert G, and Nau H (1989) Teratogenicity and placental transfer of all-trans-, 13-cis-, 4-oxo-all-trans-, and 4-oxo-13-cisretinoic acid after administration of a low oral dose during organogenesis in mice. Toxicol Appl Pharmacol 100:162-176.

Duester G (2008) Retinoic acid synthesis and signaling during early organogenesis. Cell 134:921-931.

Gaemers IC, van Pelt AM, van der Saag PT, and de Rooij DG (1996) All-trans-4-oxoretinoic acid: a potent inducer of in vivo proliferation of growth-arrested A spermatogonia in the vitamin A-deficient mouse testis. Endocrinology 137:479-485.

Gudas LJ (2012) Emerging roles for retinoids in regeneration and differentiation in normal and disease states. Biochim Biophys Acta 1821:213-221.

Gudas LJ and Wagner JA (2011) Retinoids regulate stem cell differentiation. J Cell Physiol 226:322-330.

Herrmann K (1995) Teratogenic effects of retinoic acid and related substances on the early development of the zebrafish (Brachydanio rerio) as assessed by a novel scoring system. Toxicol In Vitro 9:267-283.

Hogarth CA and Griswold MD (2013) Retinoic acid regulation of male meiosis. Curr Opin Endocrinol Diabetes Obes 20:217-223.

Idres N, Marill J, Flexor MA, and Chabot GG (2002) Activation of retinoic acid receptor-dependent transcription by all-trans-retinoic acid metabolites and isomers. J Biol Chem 277:31491-31498.

Kane MA, Folias AE, Pingitore A, Perri M, Obrochta KM, Krois CR, Cione E, Ryu JY, and Napoli JL (2010) Identification of 9-cis-retinoic acid as a pancreas-specific autacoid that attenuates glucose-stimulated insulin secretion. Proc Natl Acad Sci USA 107:21884-21889.

Kane MA, Folias AE, Wang C, and Napoli JL (2008) Quantitative profiling of endogenous retinoic acid in vivo and in vitro by tandem mass spectrometry. Anal Chem 80:1702-1708.

Kewn S, Hoggard PG, Sales SD, Johnson MA, and Back DJ (2000) The intracellular activation of lamivudine (3TC) and determination of $2^{\prime}$-deoxycytidine-5' triphosphate (dCTP) pools in the presence and absence of various drugs in HepG2 cells. Br J Clin Pharmacol 50:597-604.

Loudig O, Maclean GA, Dore NL, Luu L, and Petkovich M (2005) Transcriptional cooperativity between distant retinoic acid response elements in regulation of Cyp26A1 inducibility. Biochem J 392:241-248.

Lutz JD, Dixit V, Yeung CK, Dickmann LJ, Zelter A, Thatcher JE, Nelson WL, and Isoherranen N (2009) Expression and functional characterization of cytochrome P450 26A1, a retinoic acid hydroxylase. Biochem Pharmacol 77:258-268.

Maden M (2007) Retinoic acid in the development, regeneration and maintenance of the nervous system. Nat Rev Neurosci 8:755-765.

McBurney MW (1993) P19 embryonal carcinoma cells. Int $J$ Dev Biol 37:135-140

Muindi J, Frankel SR, Miller WH, Jr, Jakubowski A, Scheinberg DA, Young CW, Dmitrovsky E, and Warrell RP, Jr (1992a) Continuous treatment with all-trans retinoic acid causes a progressive reduction in plasma drug concentrations: implications for relapse and retinoid "resistance" in patients with acute promyelocytic leukemia. Blood 79:299-303.

Muindi JR, Frankel SR, Huselton C, DeGrazia F, Garland WA, Young CW, and Warrell RP, Jr (1992b) Clinical pharmacology of oral all-trans retinoic acid in patients with acute promyelocytic leukemia. Cancer Res 52:2138-2142.
Muindi JR, Roth MD, Wise RA, Connett JE, O’Connor GT, Ramsdell JW, Schluger NW, Romkes M, Branch RA, and Sciurba FC; FORTE Study Investigators (2008) Pharmacokinetics and metabolism of all-trans- and 13-cis-retinoic acid in pulmonary emphysema patients. J Clin Pharmacol 48:96-107.

Napoli JL (2012) Physiological insights into all-trans-retinoic acid biosynthesis. Biochim Biophys Acta 1821:152-167.

Niederreither K, Abu-Abed S, Schuhbaur B, Petkovich M, Chambon P, and Dollé P (2002) Genetic evidence that oxidative derivatives of retinoic acid are not involved in retinoid signaling during mouse development. Nat Genet 31:84-88.

Pijnappel WW, Hendriks HF, Folkers GE, van den Brink CE, Dekker EJ, Edelenbosch C, van der Saag PT, and Durston AJ (1993) The retinoid ligand 4-oxoretinoic acid is a highly active modulator of positional specification. Nature $\mathbf{3 6 6}$ : 340-344.

Rhinn M and Dollé P (2012) Retinoic acid signalling during development. Development 139:843-858.

Roberts AB, Lamb LC, and Sporn MB (1980) Metabolism of all-trans-retinoic acid in hamster liver microsomes: oxidation of 4-hydroxy- to 4-keto-retinoic acid. Arch Biochem Biophys 199:374-383.

Samokyszyn VM, Gall WE, Zawada G, Freyaldenhoven MA, Chen G, Mackenzie PI, Tephly TR, and Radominska-Pandya A (2000) 4-hydroxyretinoic acid, a novel substrate for human liver microsomal UDP-glucuronosyltransferase(s) and recombinant UGT2B7. J Biol Chem 275:6908-6914.

Schug TT, Berry DC, Shaw NS, Travis SN, and Noy N (2007) Opposing effects of retinoic acid on cell growth result from alternate activation of two different nuclear receptors. Cell 129:723-733.

Shimshoni JA, Roberts AG, Scian M, Topletz AR, Blankert SA, Halpert JR, Nelson WL, and Isoherranen N (2012) Stereoselective formation and metabolism of 4-hydroxy-retinoic Acid enantiomers by cytochrome p450 enzymes. J Biol Chem 287:42223-42232.

Tay S, Dickmann L, Dixit V, and Isoherranen N (2010) A comparison of the roles of peroxisome proliferator-activated receptor and retinoic acid receptor on CYP26 regulation. Mol Pharmacol 77:218-227.

Thatcher JE, Buttrick B, Shaffer SA, Shimshoni JA, Goodlett DR, Nelson WL, and Isoherranen N (2011) Substrate specificity and ligand interactions of CYP26A1, the human liver retinoic acid hydroxylase. Mol Pharmacol 80:228-239.

Thatcher JE, Zelter A, and Isoherranen N (2010) The relative importance of CYP26A1 in hepatic clearance of all-trans retinoic acid. Biochem Pharmacol 80:903-912.

Topletz AR, Thatcher JE, Zelter A, Lutz JD, Tay S, Nelson WL, and Isoherranen N (2012) Comparison of the function and expression of CYP26A1 and CYP26B1, the two retinoic acid hydroxylases. Biochem Pharmacol 83:149-163.

Wang T, Ma X, Krausz KW, Idle JR, and Gonzalez FJ (2008) Role of pregnane X receptor in control of all-trans retinoic acid (ATRA) metabolism and its potential contribution to ATRA resistance. J Pharmacol Exp Ther 324:674-684.

White JA, Beckett-Jones B, Guo YD, Dilworth FJ, Bonasoro J, Jones G, and Petkovich M (1997) cDNA cloning of human retinoic acid-metabolizing enzyme (hP450RAI) identifies a novel family of cytochromes P450. J Biol Chem 272: 18538-18541.

White JA, Ramshaw H, Taimi M, Stangle W, Zhang A, Everingham S, Creighton S, Tam SP, Jones G, and Petkovich M (2000) Identification of the human cytochrome P450, P450RAI-2, which is predominantly expressed in the adult cerebellum and is responsible for all-trans-retinoic acid metabolism. Proc Natl Acad Sci USA 97: 6403-6408.

Yamamoto Y, Zolfaghari R, and Ross AC (2000) Regulation of CYP26 (cytochrome P450RAI) mRNA expression and retinoic acid metabolism by retinoids and dietary vitamin A in liver of mice and rats. FASEB $J$ 14:2119-2127.

Yu S, Levi L, Siegel R, and Noy N (2012) Retinoic acid induces neurogenesis by activating both retinoic acid receptors (RARs) and peroxisome proliferatoractivated receptor $\beta / \delta(\mathrm{PPAR} \beta / \delta)$. J Biol Chem 287:42195-42205.

Zhang Y, Zolfaghari R, and Ross AC (2010) Multiple retinoic acid response elements cooperate to enhance the inducibility of CYP26A1 gene expression in liver. Gene 464:32-43.

Address correspondence to: Nina Isoherranen, Department of Pharmaceutics, University of Washington, H-272, Box 357610, Seattle, WA 98195. E-mail: ni2@u.washington.edu 